ARTICLE

\title{
Thiocysteine lyases as polyketide synthase domains installing hydropersulfide into natural products and a hydropersulfide methyltransferase
}

\author{
Song Meng ${ }^{1,4}$, Andrew D. Steele ${ }^{1,4}$, Wei Yan ${ }^{1,4}$, Guohui Pan ${ }^{1}$, Edward Kalkreuter (1) ${ }^{1}$, Yu-Chen Liu',
} Zhengren $\mathrm{Xu}^{1} \&$ Ben Shen (1, 1,3凶

Nature forms S-S bonds by oxidizing two sulfhydryl groups, and no enzyme installing an intact hydropersulfide (-SSH) group into a natural product has been identified to date. The leinamycin (LNM) family of natural products features intact S-S bonds, and previously we reported an SH domain ( $\mathrm{LnmJ}$-SH) within the LNM hybrid nonribosomal peptide synthetase (NRPS)-polyketide synthase (PKS) assembly line as a cysteine lyase that plays a role in sulfur incorporation. Here we report the characterization of an S-adenosyl methionine (SAM)dependent hydropersulfide methyltransferase (GnmP) for guangnanmycin (GNM) biosynthesis, discovery of hydropersulfides as the nascent products of the GNM and LNM hybrid NRPS-PKS assembly lines, and revelation of three SH domains (GnmT-SH, LnmJ-SH, and WsmR-SH) within the GNM, LNM, and weishanmycin (WSM) hybrid NRPS-PKS assembly lines as thiocysteine lyases. Based on these findings, we propose a biosynthetic model for the LNM family of natural products, featuring thiocysteine lyases as PKS domains that directly install a -SSH group into the GNM, LNM, or WSM polyketide scaffold. Genome mining reveals that $\mathrm{SH}$ domains are widespread in Nature, extending beyond the LNM family of natural products. The $\mathrm{SH}$ domains could also be leveraged as biocatalysts to install an - $\mathrm{SSH}$ group into other biologically relevant scaffolds.

\footnotetext{
${ }^{1}$ Department of Chemistry, The Scripps Research Institute, 130 Scripps Way, Jupiter, FL 33458, USA. ${ }^{2}$ Department of Molecular Medicine, The Scripps Research Institute, 130 Scripps Way, Jupiter, FL 33458, USA. ${ }^{3}$ Natural Products Discovery Center at Scripps Research, The Scripps Research Institute, 130 Scripps Way, Jupiter, FL 33458, USA. ${ }^{4}$ These authors contributed equally: Song Meng, Andrew D. Steele, Wei Yan. ${ }^{凶}$ email: shenb@scripps.edu
} 
S ulfur-containing natural products are structurally diverse and biologically rich in activities. Nature's ability to incorporate sulfurs into natural products provides opportunities for chemistry and enzymology discovery ${ }^{1-5}$. Cysteine and methionine are often incorporated directly into peptide natural products, usually with limited downstream transformations (Supplementary Fig. 1a, b) ${ }^{6-9}$. The sulfur sources for many other sulfur-containing natural products cannot be readily predicted, and their incorporation often involves intricate tailoring reactions of the nascent intermediates ${ }^{10-13}$. For natural products containing disulfide bonds, the S-S bonds generally result from intramolecular or intermolecular oxidation of two sulfhydryl (-SH) groups (Supplementary Fig. 1c) ${ }^{14-19}$. To date, no biosynthetic pathway or enzyme installing an intact hydropersulfide (-SSH) group into natural products has been identified.

Leinamycin (LNM, 1) is a sulfur-containing natural product featuring a 1,3-dioxo-1,2-dithiolane moiety (i.e., containing an S-S bond), spiro-fused to an 18-membered macrolactam ring (Fig. 1a) ${ }^{20}$. We previously cloned the LNM biosynthetic gene cluster (BGC) from Streptomyces atroolivaceus S-140 and discovered a DUF-SH didomain, i.e., domain of unknown function (DUF)-cysteine lyase (SH), within the final polyketide synthase (PKS) module-8 of LnmJ of the LNM hybrid non-ribosomal peptide synthetase (NRPS)-PKS assembly line (Fig. 1b, d, and Supplementary Fig. 2a $)^{21,22}$. Isolation of LNM E1 (2, Fig. 1a), a presumed biosynthetic intermediate, from both $S$. atroolivaceus S-140 wild-type and selected mutant strains ${ }^{23}$, led to the proposal that the LNM hybrid NRPS-PKS assembly line would play a role in installing only one of the two sulfur atoms of the dioxodithiolane moiety of $\mathbf{1}$. It was hypothesized that an L-cysteinepolyketide adduct, potentially generated by the LnmJ-DUF domain, could be cleaved at the cysteinyl $\mathrm{C}-\mathrm{S}$ bond by the LnmJ-SH domain to install an -SH group at C-3 of the LNM hybrid peptide-polyketide scaffold, affording 2 as the nascent product of the LNM hybrid NRPS-PKS assembly line (Supplementary Fig. $2 b)^{21-23}$. When assayed with varying substrate mimics, LnmJ-SH indeed exhibited a cysteine lyase activity in vitro, albeit inefficiently (Supplementary Fig. 2c) ${ }^{22}$. These findings suggest that the S-S bond would have been formed by a tailoring enzyme post the NRPS-PKS assembly line catalysis. However, all attempts to identify the origin of the second sulfur atom and to establish the biosynthetic pathway from $\mathbf{2}$ to $\mathbf{1}$ have failed despite exhaustive efforts (Supplementary Fig. 2d).

To explore Nature's biosynthetic reservoir for the LNM family of natural products, we recently completed a genome mining campaign, using the LnmJ-DUF-SH didomain as a molecular beacon to identify BGCs encoding other members of the LNM family of natural products, culminating in the discovery of the guangnanmycins (GNM A, 3, and GNM B, 4) from Streptomyces sp. CB01883 and the weishanmycins (WSM A, 5 and WSM A1, 6) from Streptomyces sp. CB02120-2 (Fig. 1a, b) ${ }^{24,25}$. The structure of $\mathbf{3}$, in particular, is biosynthetically enlightening, containing a methyldisulfide moiety, a much simpler modification than the 1,3-dioxo-1,2-dithiolane moiety in $\mathbf{1}$, and featuring an intact disulfide bond unlike $2, \mathbf{4}$, and $\mathbf{5}$ that are characterized with a "-SH" group (Fig. 1a). The presence of the methyltransferase (MT) GnmP encoded within the GNM BGC further hints at the potential involvement of a hydropersulfide biosynthetic intermediate en route to 3 . Taken together, we reasoned that $\mathbf{3}$ would serve as a better model system than $\mathbf{1}$ to study disulfide incorporation in natural product biosynthesis.

Herein, we first report GnmP as $S$-adenosyl methionine (SAM)-dependent MT that exhibits a high substrate preference for GNM P (7), a hydropersulfide (Fig. 2), establishing 7 as the penultimate intermediate for the biosynthesis of 3 (Fig. 1d). We next establish $\mathbf{4}$ as the disproportionation product of $\mathbf{7}$, combined with 2 as the disproportionation product of the corresponding hydropersulfide congener LNM E (8) (Fig. 1a), revealing hydropersulfides as common biosynthetic intermediates in the biosynthesis of the LNM family of natural products (Figs. 1 and 3). We then demonstrate that GnmT-SH, LnmJ-SH, and WsmR-SH are thiocysteine lyases, rather than cysteine lyases, leading to the discovery of thiocysteine lyases as PKS domains that directly install a -SSH group into the GNM, LNM, or WSM polyketide scaffold (Fig. 4) and providing a biosynthetic model for the LNM family of natural products (Fig. 1d). We finally reveal that $\mathrm{SH}$ domains are widespread in Nature, extending beyond the LNM family of natural products (Fig. 1c), and the SH domains could be further leveraged as biocatalysts to install an -SSH group into other biologically relevant scaffolds (Fig. 5).

\section{Results and discussion}

Characterization of GnmP as a SAM-dependent MT exhibiting a high substrate preference for hydropersulfides. We first characterized GnmP as a SAM-dependent hydropersulfide MT, establishing 7 as the penultimate intermediate for the biosynthesis of 3 (Figs. 1d and 2). Comparative analysis of the $\mathrm{lnm}$ and gnm BGCs identified $g n m P$ as a candidate encoding a SAM-dependent MT; however, bioinformatics analysis fell short of predicting $\mathrm{GnmP}$ as a $\mathrm{C}$-, $\mathrm{O}-, \mathrm{N}-$, or $\mathrm{S}$-MT, as hydropersulfide $\mathrm{MT}$ is unknown to date (Supplementary Fig. 6). We inactivated gnmP in S. sp. CB01883, affording the $\triangle g n m P$ mutant strain SB21007 (Supplementary Fig. 7) that abolished production of $\mathbf{3}$, and complementation of the $\triangle g n m P$ mutation by expressing a functional copy of gnmP in trans (i.e., SB21008) restored the production of 3 (Fig. 2b). Large-scale fermentation of SB21007 followed by isolation and structural elucidation of the accumulated metabolites indeed resulted in the detection of 7 , albeit in trace quantities (Supplementary Fig. 8a, b), with the major metabolites, including the thiol $\mathbf{4}$, isolated and characterized as degradation products of 7 due to its intrinsic instability (Supplementary Figs. 8c, 9, and 10).

We developed a chemoenzymatic method to generate the labile hydropersulfides in situ and confirmed GnmP as a SAMdependent hydropersulfide MT by directly assaying its activity in vitro. GnmP was readily overproduced in Escherichia coli and purified (Supplementary Fig. 11). However, all attempts to isolate 7 directly from SB21007 or prepare 7 semisynthetically from $\mathbf{4}$ as the substrate for GnmP failed due to its intrinsic instability. We subsequently resorted to a chemoenzymatic method to generate 7 in situ, leveraging the cystine lyase activity of LnmJ-SH reported previously (Fig. 2a) 22 . Thus, by following the same protocol established previously for LnmJ-SH, we overproduced GnmT-SH, LnmJ-SH, and WsmR-SH in E. coli and purified them to homogeneity (Supplementary Fig. 12) 22 . We prepared the Lcysteinyl-GNM B adduct (9) from 4 semisynthetically (Supplementary Fig. 13). Treatment of 9 with GnmT-SH produced 7 in situ, the rapid formation of which can be quantitively followed with the thiol-trapping reagent monobromobimane $(\mathrm{mBB}, 10)$ (Fig. 2c) and confirmed by structural characterization of the resultant $\mathrm{mBB}$ adduct (11) (Supplementary Fig. 10). When 9 was treated with GnmT-SH, in the presence of GnmP and SAM, a concentration-dependent consumption of 9 was observed with concomitant production of $\mathbf{3}$, the identity of which was established by comparison with an authentic standard (Fig. 2c).

We determined the strong substrate preference of GnmP for 7 over its thiol congener $\mathbf{4}$ by directly comparing its kinetic parameters with $\mathbf{7}$ or $\mathbf{4}$ as a substrate. We first showed that the three SH domains were all catalytically competent (Supplementary Fig. 14) and, under the optimized assay conditions, GnmT$\mathrm{SH}, \mathrm{LnmJ}-\mathrm{SH}$, or WsmR-SH-catalyzed production of 7 from 9 
a

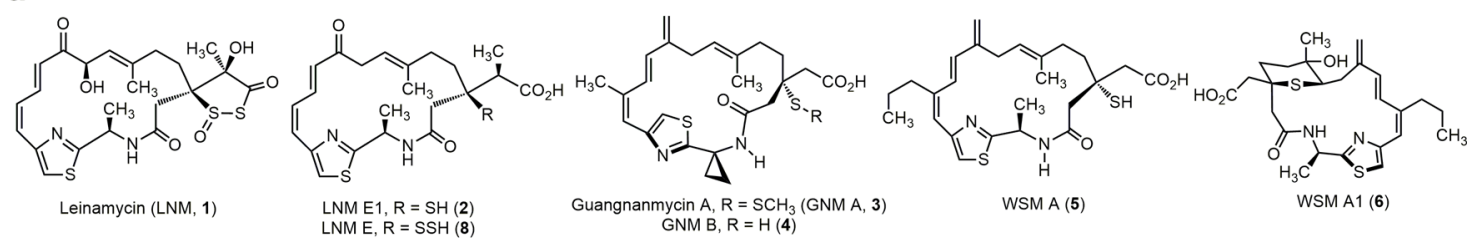

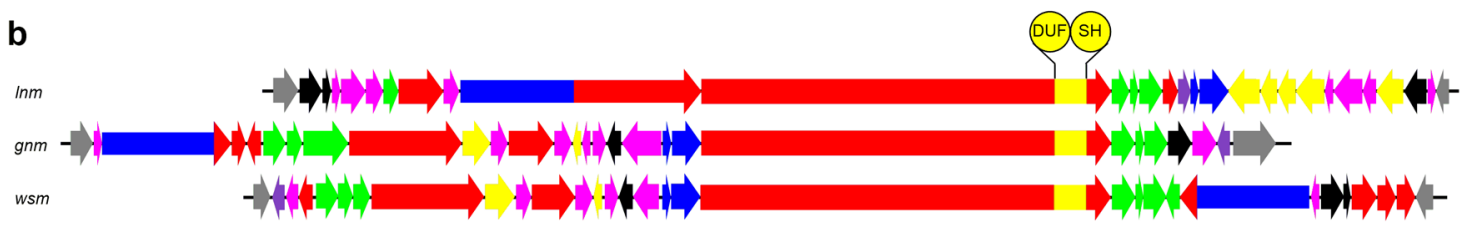

C
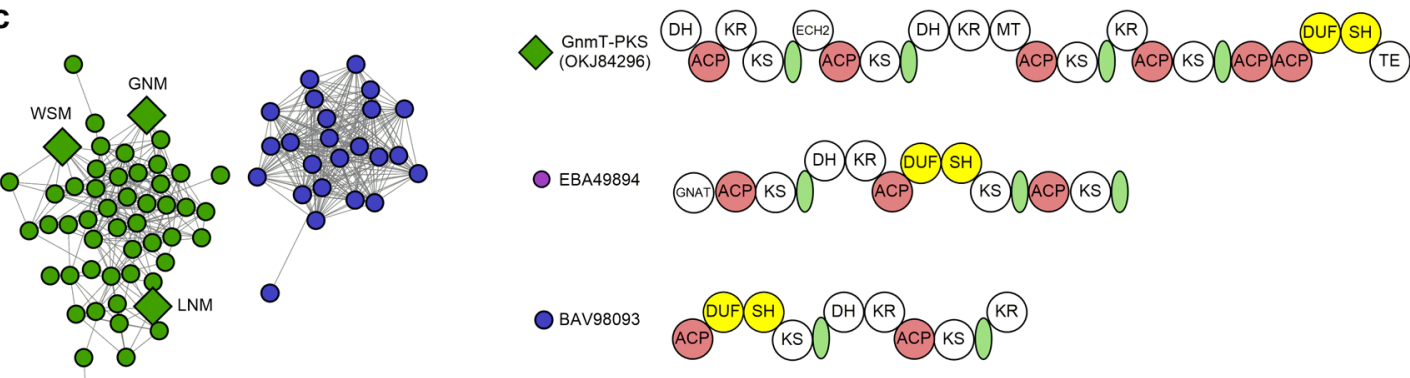

O EBA49894

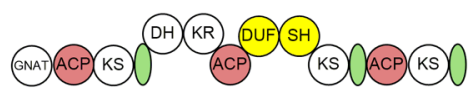

BAV98093
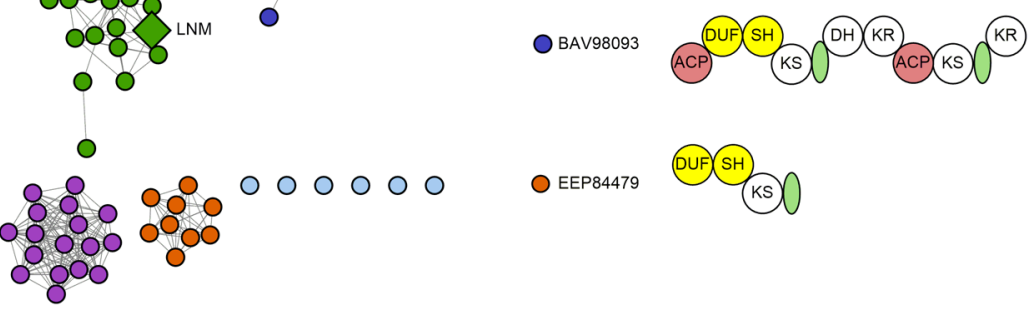

OEP84479
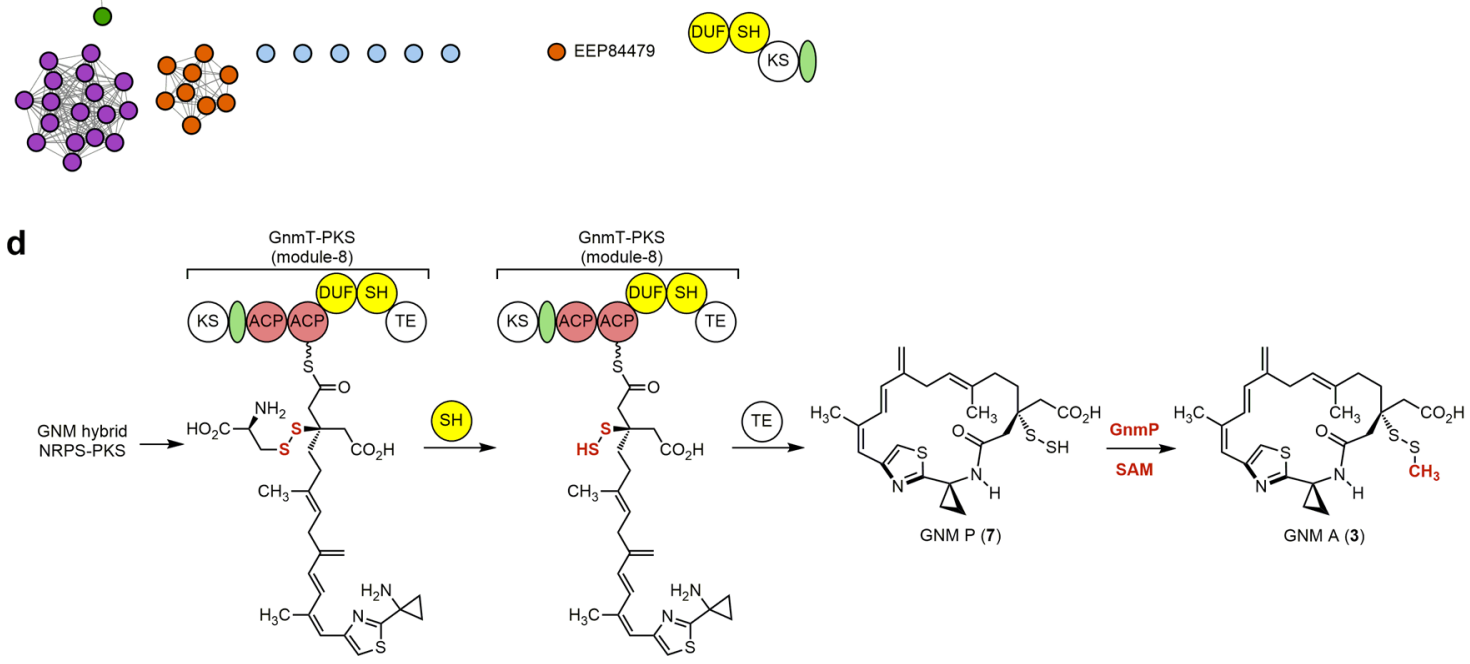

Fig. 1 GNM biosynthesis as a model for the LNM family of natural products featuring a thiocysteine lyase as a PKS domain that directly installs an -SSH group into the GNM hybrid peptide-polyketide scaffold. a The structures of LNM (1), GNM A (3), and congeners LNM E1 (2), LNM E (8), GNM B (4), WSM A (5), and WSM A1 (6) (Supplementary Fig. 3). b The Inm, gnm, and wsm BGCs featuring DUF-SH didomain-containing type I PKSs. c Sequence similarity network (SSN) of 109 DUF-SH domain-containing PKS proteins revealing four major clusters, with representative PKS modular architectures shown for the four major clusters (see Supplementary Figs. 4 and 5 for a complete summary). The DUF-SH didomain-containing PKS proteins from the LNM-type BGCs are color-coded in green, with the corresponding PKSs from the LNM, GNM, and WSM BGCs depicted as diamonds. d The biosynthetic pathway of $\mathbf{3}$ highlighting (i) GnmT-SH domain-catalyzed installation of the -SSH group via an L-thiocysteine adduct at C-3 of the growing polyketide chain, (ii) hydropersulfide $\mathbf{7}$ as the nascent product of the GNM hybrid NRPS-PKS assembly line, and (iii) GnmP-catalyzed S-methylation of $\mathbf{7}$ to afford $\mathbf{3}$. ACP acyl carrier protein, DH dehydratase, DUF domain of the unknown function, ECH2 enoyl-CoA hydratase homolog 2, GNAT GCN5-related Nacetyltransferase, KR ketoreductase, KS ketosynthase, MT methyltransferase, SH thiocysteine lyase, TE thioesterase, and the green ovals denoting an acyltransferase docking domain.

followed Michaelis-Menten kinetics ${ }^{22}$. By comparing their steady-state kinetic parameters (Supplementary Fig. 15), we revealed that WsmR-SH exhibited the highest catalytic efficiency $\left(k_{\text {cat }} / K_{\mathrm{m}}\right)$ in generating 7 from $\mathbf{9}$, among the three SH domains tested. This allowed us to develop a WsmR-SH and GnmPcoupled assay for kinetic analysis of $\mathrm{GnmP}$ using $\mathbf{9}$ as a surrogate substrate to circumvent the intrinsic instability of 7 (Supplementary Fig. 16a). By generating 7 in situ using an excess of WsmR-
SH $(220 \mu \mathrm{M})$ relative to $\mathrm{GnmP}(50 \mathrm{nM})$, we showed that GnmPcatalyzed formation of $\mathbf{3}$ followed Michaelis-Menten kinetics and determined the steady-state kinetic parameters, with a $K_{\mathrm{m}}$ value of $2.45 \pm 0.41 \mu \mathrm{M}$ and a $k_{\text {cat }}$ value of $9.53 \pm 0.36 \mathrm{~min}^{-1}$ (Supplementary Fig. 16b). GnmP could also catalyze $S$-methylation of 4, but very inefficiently (Supplementary Figs. 16c, d, e, and 17a), and steady-state kinetic parameters of GnmP-catalyzed $S$-methylation of 4 were similarly determined, with a $K_{\mathrm{m}}$ value of $12.1 \pm 2.2 \mu \mathrm{M}$ 

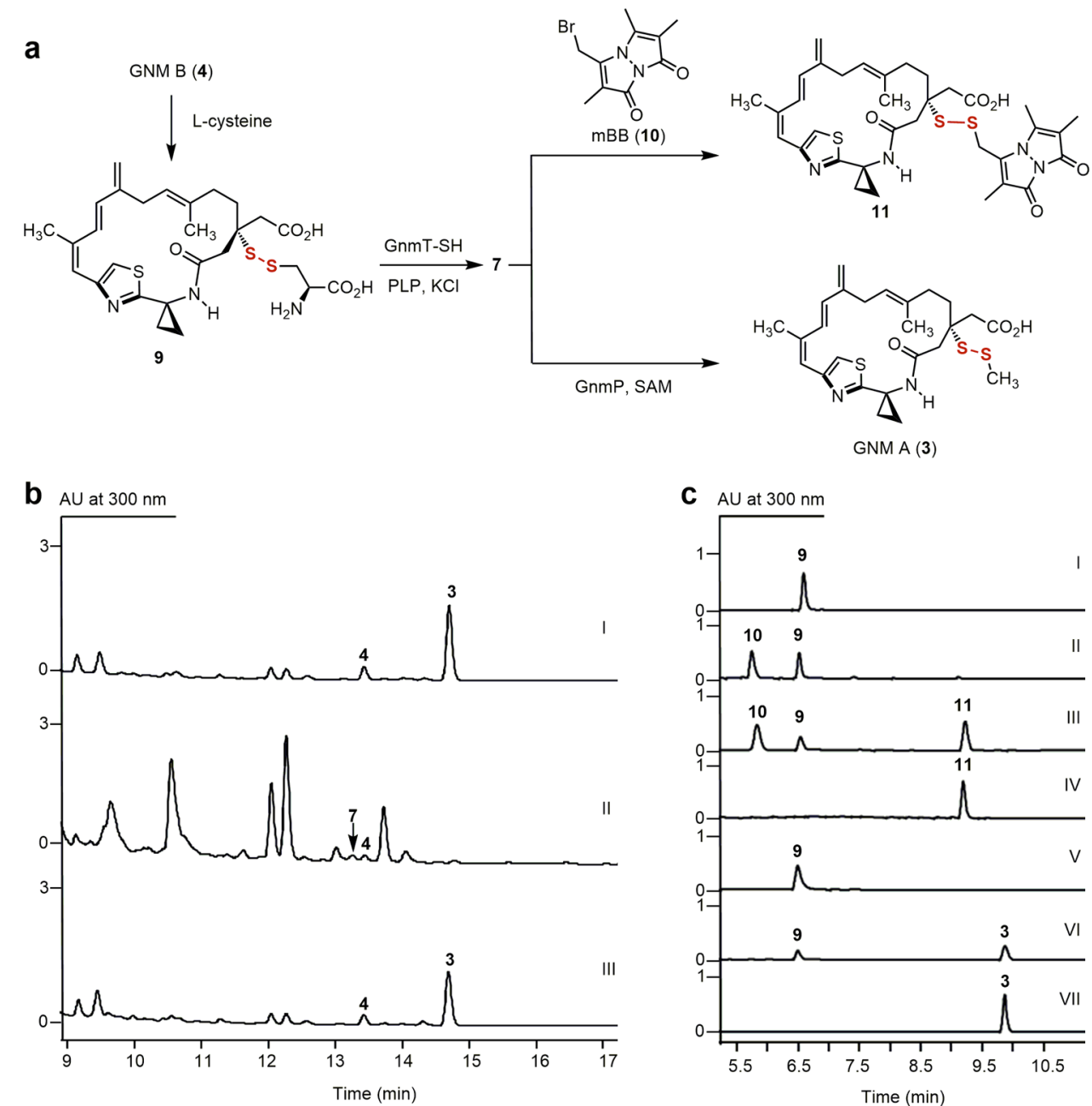

Fig. 2 In vivo and in vitro characterization of GnmP as a SAM-dependent hydropersulfide methyltransferase, establishing 7 as the penultimate intermediate for $\mathbf{3}$ biosynthesis. a Chemoenzymatic synthesis of $\mathbf{7}$ from $\mathbf{4}$ and a GnmT-SH and GnmP-coupled assay of GnmP-catalyzed S-methylation of $\mathbf{7}$ to $\mathbf{3}$ using $\mathbf{9}$ as a surrogate substrate. b HPLC analysis of metabolite profiles: (I) S. sp. CB01883 (wild-type), (II) SB21007 ( $\Delta$ gnmP), and (III) SB21008 $(\Delta g n m P / g n m P)$. c HPLC analysis of in vitro assays: (I) substrate $\mathbf{9}$, (II) $\mathbf{9}+\mathrm{GnmT}-\mathrm{SH}$ (boiled) + 10, (III) $\mathbf{9}+\mathrm{GnmT}$-SH $+\mathbf{1 0}$, (IV) product 11, (V) $\mathbf{9}+\mathrm{GnmT}-\mathrm{SH}$ (boiled) + GnmP (boiled) + SAM, (VI) $9+\mathrm{GnmT}-\mathrm{SH}+\mathrm{GnmP}+\mathrm{SAM}$, and (VII) product 3. Substrate and enzyme concentrations used: 9 , $0.5 \mathrm{mM}$; SAM, $2 \mathrm{mM}$; GnmT-SH, $10 \mu \mathrm{M}$; and GnmP, $30 \mu \mathrm{M}$. Incubation time: 20 min. The concentration of $\mathbf{1 0}$ used to trap $\mathbf{7}$ in situ as $\mathbf{1 1 :} 0.5 \mathrm{mM}$. "AU" denotes absorbance units.

and a $k_{\text {cat }}$ value of $(4.36 \pm 0.29) \times 10^{-3} \mathrm{~min}^{-1}$ (Supplementary Fig. 16f). GnmP exhibits more than 10,000-fold higher catalytic efficiency for $7\left(k_{\mathrm{cat}} / K_{\mathrm{m}}=3.9 \mu \mathrm{M}^{-1} \mathrm{~min}^{-1}\right)$ over $4\left(k_{\mathrm{cat}} / K_{\mathrm{m}}=\right.$ $\left.3.6 \times 10^{-4} \mu \mathrm{M}^{-1} \mathrm{~min}^{-1}\right)$, in agreement with the in vivo results that support 7 as the penultimate intermediate for the biosynthesis of $\mathbf{3}$ (Fig. 2c).

Establishment of hydropersulfide intermediates in the biosynthesis of the LNM family of natural products. The finding of GnmP as a SAM-dependent hydropersulfide MT in the biosynthesis of 3 , combined with our inability to experimentally verify 2 as an intermediate in LNM biosynthesis (i.e., conversion of $\mathbf{2}$ to 1, Supplementary Fig. 2d), prompted us to investigate hydropersulfides as common intermediates in the biosynthesis of the LNM family of natural products. Since hydropersulfides are known to undergo disproportionation, affording a thiol and a hydrotrisulfide that can undergo further decomposition ${ }^{26}$, the intrinsic instability of 7 promoted us to ask if $\mathbf{4}$ is a disproportionation product of 7 , rather than a true intermediate, in the biosynthesis of $\mathbf{3}$. In situ generation of 7 by treating 9 with an excess of WsmR-SH indeed resulted in detectable quantities of $\mathbf{4}$, and when this experiment was repeated with the addition of GnmP and SAM, the $S$-methylated trisulfide product (12) of 4 was also observed (Fig. 3a, b and Supplementary Fig. 17b). The Lcysteinyl-LNM E1 adduct (13) was then similarly prepared from $\mathbf{2}$ as a substrate to prepare the corresponding hydropersulfide $\mathbf{8}$ chemoenzymatically. Treatment of $\mathbf{1 3}$ with an excess of WsmR$\mathrm{SH}$ produced 8 in situ, the identity of which was confirmed by structural characterization of its $\mathrm{mBB}$ adduct (Supplementary Figs. 10 and $18 \mathrm{a}, \mathrm{b})$. When the same experiment was repeated with the addition of GnmP and SAM, the S-methylated trisulfide product (15) was indeed observed, together with 2 , as well as the $S$-methylated disulfide product (14) of 8 (Fig. 3a, c and Supplementary Figs. 10 and $18 \mathrm{a}, \mathrm{c}$ ).

The establishment of 7 as the penultimate intermediate for the biosynthesis of $\mathbf{3}$, together with the revelation of $\mathbf{2}$ and $\mathbf{4}$ as the disproportionation products of their corresponding true hydropersulfide intermediates in the biosynthesis of $\mathbf{1}$ and $\mathbf{3}$, led to the proposal of 7 , and hydropersulfides in general, as the nascent products of the hybrid NRPS-PKS assembly lines for the LNM family of natural products. These findings account for our failed attempts to date to identify the origin of the second sulfur atom 


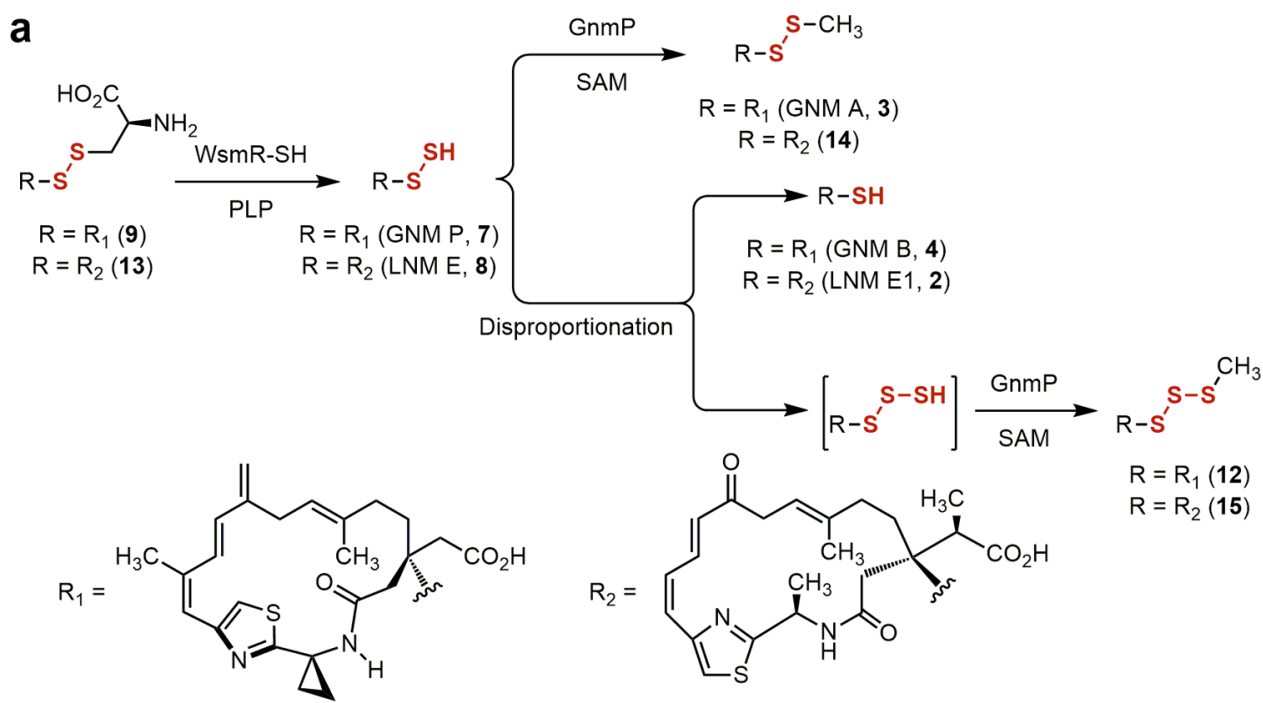

b

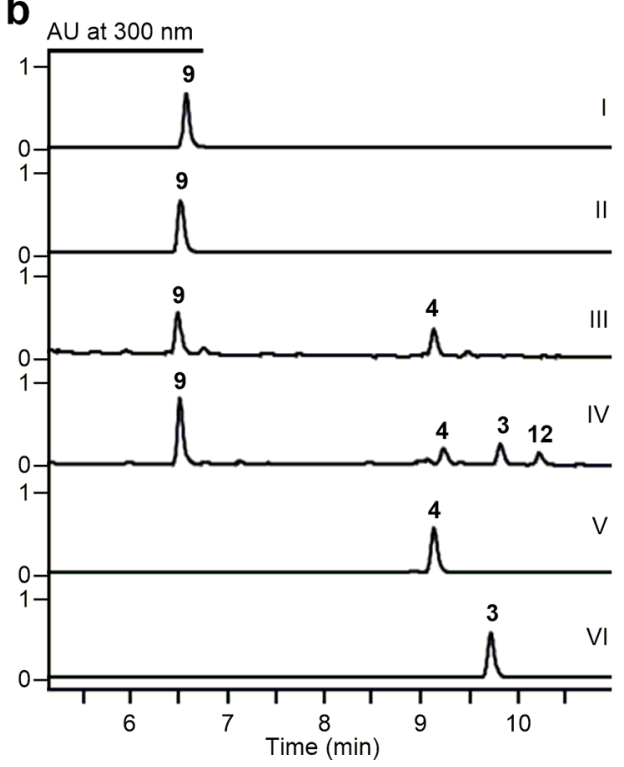

C

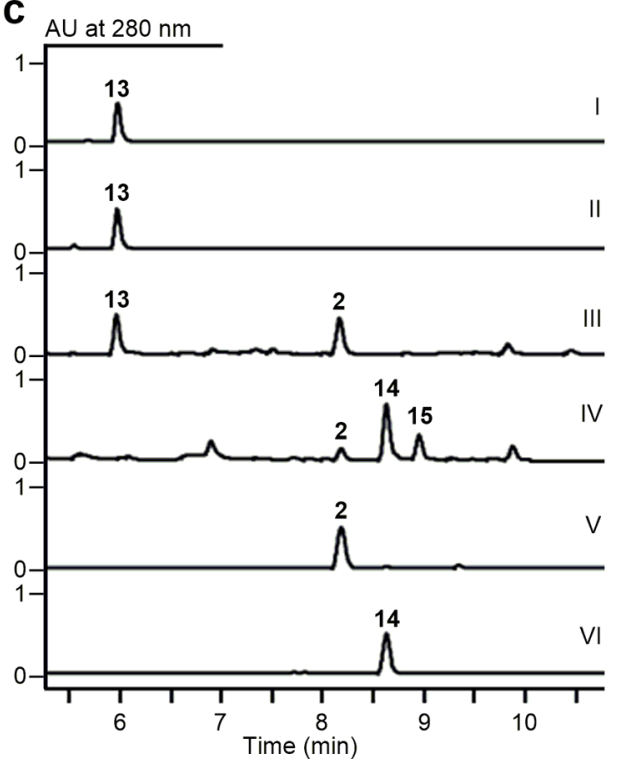

Fig. 3 Disproportionation of GNM P (7) and LNM E (8) to GNM B (4) and LNM E1 (2), and their corresponding hydrotrisulfides, revealing 4 and 2 as shunt metabolites in GNM and LNM biosynthesis. a Disproportionation reaction of $\mathbf{7}$ or $\mathbf{8}$, generated in situ using $\mathbf{9}$ or $\mathbf{1 3}$ as a surrogate substrate, to $\mathbf{4}$ or $\mathbf{2}$ and the corresponding hydrotrisulfides, which can be trapped by GnmP in situ to afford the S-methylated trisulfide $\mathbf{1 2}$ or $\mathbf{1 5}$, respectively. $\mathbf{b}$ HPLC analysis of $\mathbf{7}$ disproportionation: (I) substrate $\mathbf{9}$, (II) $\mathbf{9}+\mathrm{WsmR}-\mathrm{SH}$ (boiled), (III) $\mathbf{9}+\mathrm{WsmR}-\mathrm{SH}$, (IV) $\mathbf{9}+\mathrm{WsmR}-\mathrm{SH}+\mathrm{GnmP}+\mathrm{SAM}$, (V) standard 4, and (VI) standard 3. Substrate and enzyme concentrations used: $\mathbf{9}, 1 \mathrm{mM} ; \mathrm{SAM}, 2 \mathrm{mM} ; \mathrm{WsmR}-\mathrm{SH}, 220 \mu \mathrm{M}$; and GnmP, $10 \mu \mathrm{M}$. Incubation time: 3 min for all assays. c HPLC analysis of $\mathbf{8}$ disproportionation: (I) substrate 13, (II) $\mathbf{1 3}+$ WsmR-SH (boiled), (III) $\mathbf{1 3}+$ WsmR-SH, (IV) $\mathbf{1 3}+\mathrm{WsmR}-\mathrm{SH}+\mathrm{GnmP}+\mathrm{SAM}$, (V) standard 2, and (VI) standard 14. Substrate and enzyme concentrations used: 13, 5 mM, SAM, 10 mM; WsmR-SH, $220 \mu M$; and GnmP, $10 \mu M$. Incubation time: 3 min for assays II and III and $2 \mathrm{~h}$ for assay IV, respectively.

and to establish the intermediacy of $\mathbf{2}$ in $\mathbf{1}$ biosynthesis. Isolation of 2 was the major motivation in previous studies that led to the characterization of the $\mathrm{LnmJ}-\mathrm{SH}$ domain as a cysteine lyase installing the $-\mathrm{SH}$ group at $\mathrm{C}-3$ of the LNM hybrid peptide-polyketide scaffold (Supplementary Fig. 2b-d). Demonstration of $\mathbf{2}$ and $\mathbf{4}$ as disproportionation products of 8 and $\mathbf{7}$ in the biosynthesis of $\mathbf{1}$ and $\mathbf{3}$, respectively, therefore, inspired us to consider hydropersulfides as the nascent products of the GNM, LNM, and WSM hybrid NRPS-PKS assembly line and reevaluate the mechanism by which the -SSH groups are installed into the varying hybrid peptide-polyketide scaffolds (Fig. 1d). These findings highlight the value of GNM as a preferred model system to study disulfide incorporation in natural product biosynthesis.
The revelation of $\mathrm{SH}$ domains as thiocysteine lyases directly installing an - $\mathrm{SSH}$ group into varying polyketide scaffolds. We showed that GnmT-SH, LnmJ-SH, and WsmR-SH, the three SH domains from the GNM, LNM, and WSM biosynthetic machinery of the LNM family of natural products, uniformly act as thiocysteine lyases by direct assay of varying substrate mimics in vitro. Thus, in an analogy to our previous effort to assay the LnmJ-SH domain as a cysteine lyase using an $\mathrm{N}$-acetylcysteamine thioester (SNAC) of a truncated L-cysteine-polyketide adduct (16) as a substrate mimic ${ }^{22}$, we chemically synthesized the SNAC of a truncated L-thiocysteine-polyketide adduct (17) as a substrate mimic to the ACP-tethered growing hybrid peptide-polyketide intermediate (Fig. 1d) and directly assayed the three $\mathrm{SH}$ domains for the thiocysteine lyase activity in vitro, with $\mathbf{1 6}$ as a control to 

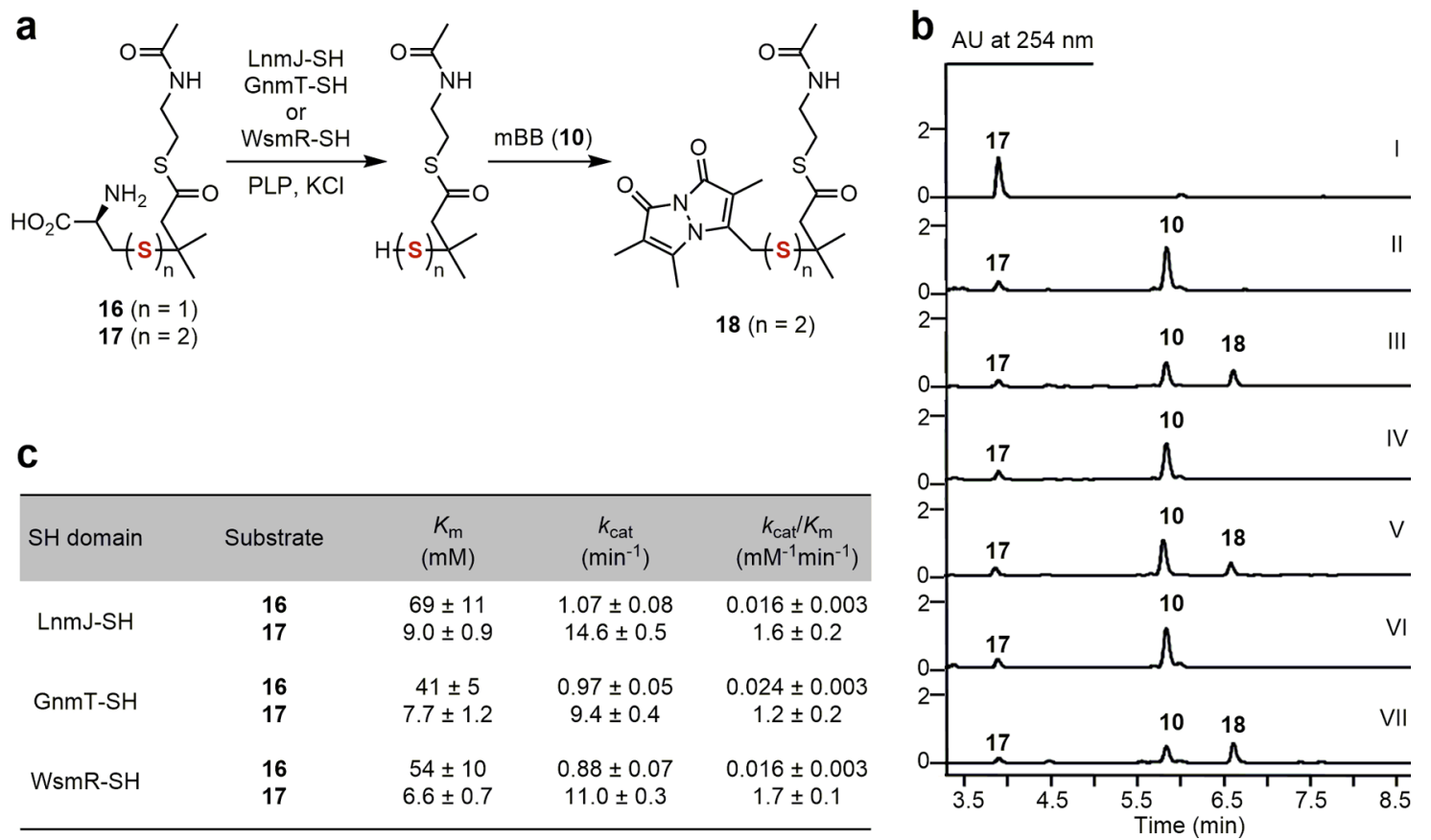

Fig. 4 Characterization of the GnmT-SH, LnmJ-SH, and WsmR-SH domains as thiocysteine lyases installing an -SSH group into varying polyketide scaffolds. a In vitro assay of the three SH domains as thiocysteine lyases using $\mathbf{1 7}$ as a substrate mimic, in comparison with $\mathbf{1 6}$ as a control for the cysteine lyase activity reported previously for LnmJ-SH. b HPLC analysis of in vitro assays: (I) substrate 17, (II) $17+\mathrm{GnmT}$-SH (boiled) + 10, (III) 17 + GnmT$\mathrm{SH}+10$, (IV) $17+$ LnmJ-SH (boiled) + 10, (V) $17+$ LnmJ-SH + 10, (VI) $17+$ WsmR-SH (boiled) + 10, (VII) $17+$ WsmR-SH (boiled) + 10, and (VIII) $17+$ WsmR-SH + 10. Substrate and enzyme concentrations used: 17, $5 \mathrm{mM} ; \mathrm{GnmT}-\mathrm{SH}$, LnmJ-SH, or WsmR-SH, $30 \mu \mathrm{M}$. Incubation time: 30 min. The concentration of $\mathbf{1 0}$ used to trap the resultant hydropersulfide product in situ as 18: $2 \mathrm{mM}$. c Comparison of the steady-state kinetic parameters of the three SH domains for $\mathbf{1 7}$ over $\mathbf{1 6}$.

compare with the cysteine lyase activity reported previously for LnmJ-SH (Fig. 4a) (Supplementary Fig. 19) ${ }^{22}$.

GnmT-SH, LnmJ-SH, and WsmR-SH indeed catalyzed cleavage of $\mathbf{1 7}$ to afford the corresponding hydropersulfide product, the identity of which was established through capture and characterization of the mBB-adduct 18 (Fig. $4 \mathrm{a}, \mathrm{b}$ ). Under the optimized conditions ${ }^{22}$, we showed that both the cysteine lyase (towards 16) and the thiocysteine lyase (towards 17) activities of the three SH domains followed Michaelis-Menten kinetics and determined their steady-state kinetic parameters. GnmT-SH, LnmJ-SH, and WsmR-SH display uniformly smaller $K_{\mathrm{m}}$ values and higher $k_{\text {cat }}$ values for 17 and exhibit 50- to 106-fold higher catalytic efficiencies $\left(k_{\mathrm{cat}} / K_{\mathrm{m}} \mathrm{s}\right)$ for 17 over 16 (Fig. 3c) (Supplementary Fig. 20). Taken together, these findings support the three $\mathrm{SH}$ domains natively functioning as thiocysteine lyases that directly install an -SSH group into the GNM, LNM, and WSM polyketide scaffolds (Fig. 1d and Supplementary Fig. 21) ${ }^{24}$.

The thiocysteine lyase activity of the SH domains, acting on the growing ACP-tethered L-thiocysteine-polyketide adduct as a substrate, as exemplified by the proposed biosynthetic pathway for 3 (Fig. 1d) (also see Supplementary Fig. 21), requires that Lthiocysteine be available as a biosynthetic precursor for the GNM, LNM, and WSM hybrid NRPS-PKS assembly lines. One established biochemical pathway to L-thiocysteine in the primary metabolism of Streptomyces is via the $\beta$-elimination reaction of $\mathrm{L}$ cystine catalyzed by cystathionine gamma lyase $(\mathrm{CGL})^{27}$. We cloned and overproduced in E. coli the three homologous CGL enzymes from the LNM-, GNM-, and WSM-producing strains $S$. sp. CB01635, S. sp. CB01883, and S. sp. CB02120-2, respectively (Supplementary Fig. 22) ${ }^{22}$. The L-cystine lyase activity of the CGL enzymes was confirmed (Supplementary Fig. 23), demonstrating that L-thiocysteine is available in S. sp. CB01635, S. sp. CB01883, and S. sp. CB02120-2 to support LNM, GNM, and WSM biosynthesis. In addition to L-thiocysteine, the deacetylated persulfide form of mycothiol, the primary thiol in Streptomyces ${ }^{28}$, was also considered as a potential persulfide donor for the $\mathrm{SH}$ domain. While mycothiol contains an $\mathrm{N}$-acetylated cysteine moiety that would preclude it from serving as a substrate for the $\mathrm{SH}$ domains by the PLP-dependent mechanism, the corresponding free amine is a known biosynthetic intermediate ${ }^{29}$. We, therefore, synthesized both the deacetylated mycothiol persulfide adduct and its monosaccharide analog, and assayed them as substrate mimics to undergo $\mathrm{SH}$-catalyzed $\mathrm{C}-\mathrm{S}$ bond cleavage in an analogous fashion to $\mathbf{1 7}$ in the presence of $\mathrm{mBB}$ (10) (Supplementary Fig. 24). However, no mBB-adduct 18 was observed under all assay conditions tested, ruling out mycothiol or its biosynthetic intermediates as potential persulfide donors and supporting an ACP-tethered L-thiocysteine-polyketide adduct as a substrate for the $\mathrm{SH}$ domains.

DUF-SH didomain-containing BGCs widely spread in Nature that extends beyond the biosynthetic machinery for the LNM family of natural products. Previously using the LnmJ-DUF-SH didomain as a molecular beacon to mine the genomes available from the public databases (as of March 2017), together with a genome survey of 5000 actinobacteria strains from the microbial strain collection at The Scripps Research Institute, we identified 49 BGCs predicted to encode 18 distinct members of the LNM family of natural products, from which the GNMs and WSMs were discovered ${ }^{24}$. Inspired by the current findings of the GnmT$\mathrm{SH}, \mathrm{LnmJ}-\mathrm{SH}$, and WsmR-SH domains as thiocysteine lyases, we re-examined the genomes available from the public databases. Among the total of 109 dereplicated BGCs (as of April 2021), we identified three additional families of BGCs encoding DUF-SH didomain-containing PKS proteins that are distinct from the BGCs encoding the LNM family of natural products (Fig. 1c). 

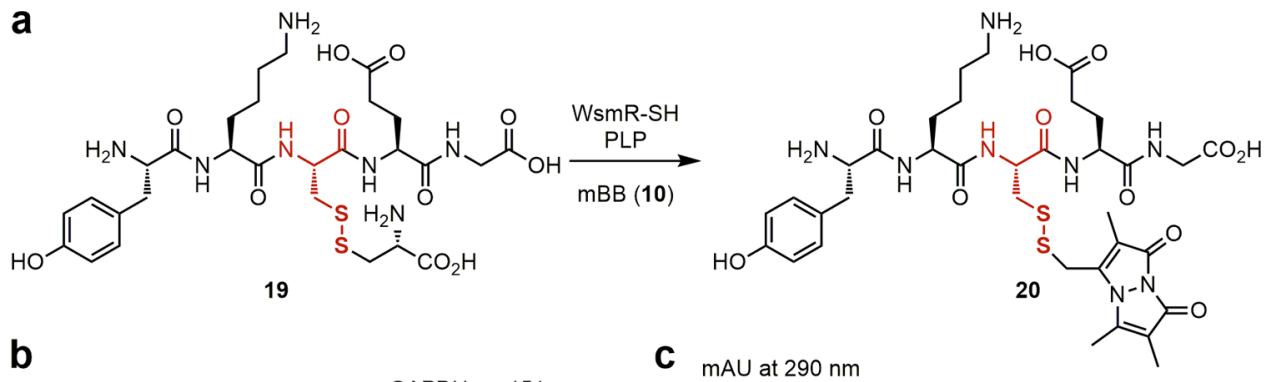

b
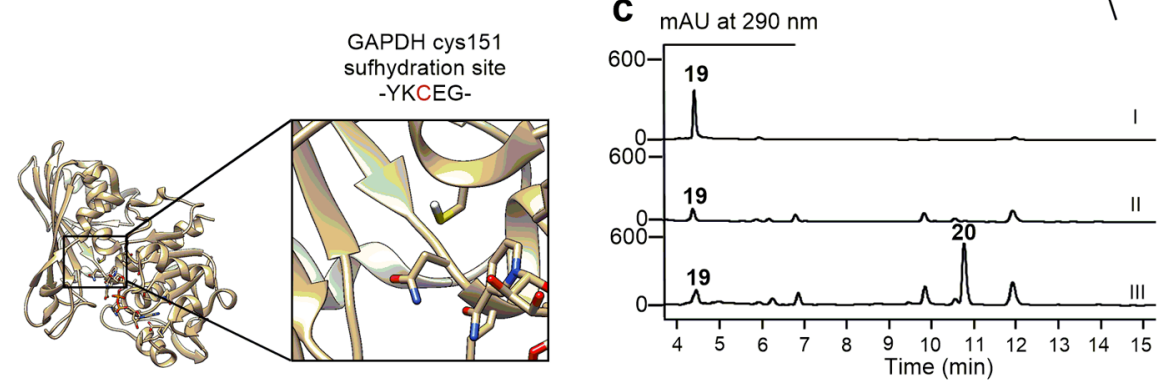

d
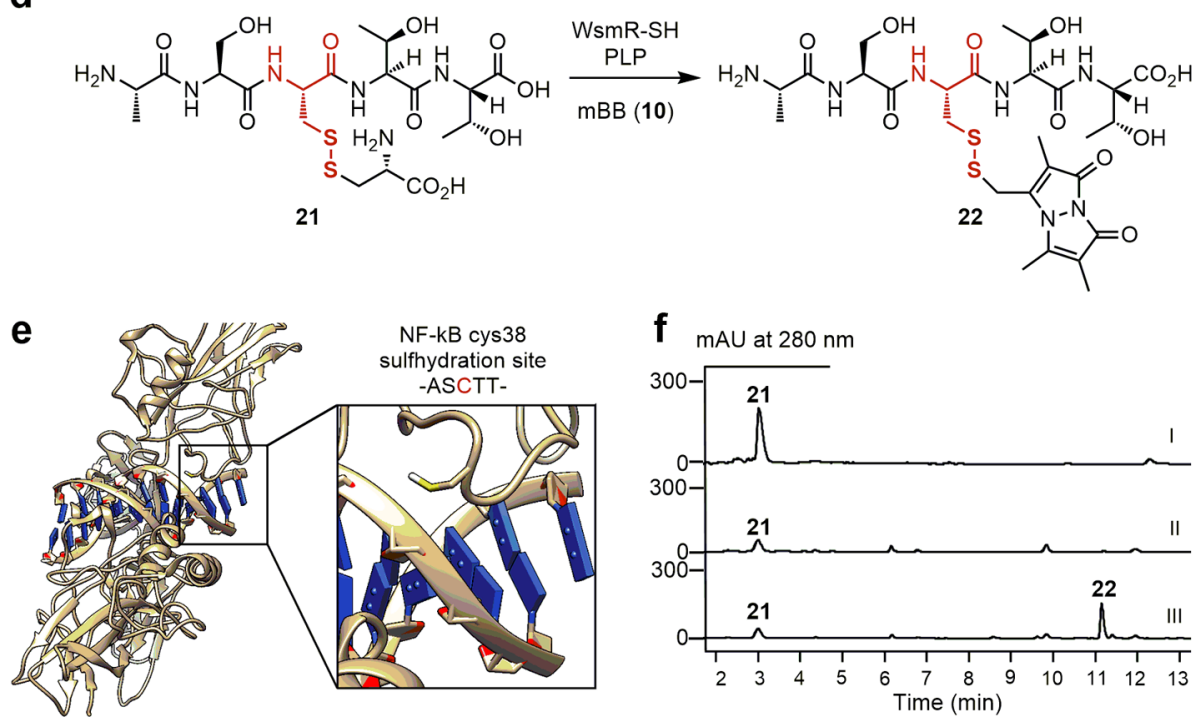

Fig. 5 Leveraging the SH domains as biocatalysts to install an -SSH group into varying peptide scaffolds. WsmR-SH-catalyzed synthesis of a 20 and $\mathbf{d}$ $\mathbf{2 2}$ from the L-thiocysteine-pentapeptide adducts $\mathbf{1 9}$ and $\mathbf{2 1}$ as substrate mimics for GAPDH and NF-kB, respectively. The crystal structures of $\mathbf{b}$ GAPDH (PDB: 1ZNQ) and NF-kB (PDB: 1LE9) highlighting the Cys151 and Cys38 residues that undergo sulfhydration, respectively. HPLC analysis of in vitro assays of c (I) substrate 19, (II) $19+\mathrm{WsmR}-\mathrm{SH}$ (boiled) + 10, and (III) $\mathbf{1 9}+\mathrm{WmR}-\mathrm{SH}+\mathbf{1 0}$ and $\mathbf{f}$, (I) substrate 21, (II) $\mathbf{2 1}+\mathrm{WsmR}-\mathrm{SH}$ (boiled) $+\mathbf{1 0}$, and (III) $\mathbf{2 1}+\mathrm{WmR}-\mathrm{SH}+\mathbf{1 0}$. Substrate and enzyme concentrations used: $\mathbf{1 9}$ or $\mathbf{2 1}, 2 \mathrm{mM}$; and WsmR-SH, $30 \mu \mathrm{M}$. Incubation time: 30 min. The concentration of $\mathbf{1 0}$ used to trap the resultant hydropersulfide product in situ as $\mathbf{2 0}$ or $\mathbf{2 2}$, respectively: $2 \mathrm{mM}$.

While the DUF-SH didomains from both the predicted LNMtype and non-LNM type BGCs are highly homologous (Supplementary Fig. 4), the PKSs harboring the DUF-SH didomains are highly variable in their modular architecture among the varying BGCs (Fig. 1c and Supplementary Fig. 5), indicative of the biosynthesis of diverse polyketide scaffolds. These findings suggest that the SH domain chemistry discovered in the context of the biosynthesis of GNM, LNM, and WSM extends beyond the LNM family of natural products. Therefore, the DUF-SH didomains appear to be genetically programmable within type I PKS assembly lines, with the $\mathrm{SH}$ domain acting as a thiocysteine lyase to install an -SSH group into varying polyketide scaffolds likely through the similar chemistry as established in this study.

Demonstration of the SH domain as a biocatalyst to install an -SSH group into peptide scaffolds. Inspired by the substrate promiscuity of the $\mathrm{SH}$ domains as thiocysteine lyases, we selected the WsmR-SH domain as a biocatalyst and demonstrated its ability to install an -SSH group into biologically relevant peptide scaffolds (Fig. 5). Hydropersulfides have emerged as an important protein post-translational modification (PTM), which are generated via the sulfhydration of cysteine residues ${ }^{30}$. Their roles in cellular signaling and redox biology have been increasingly appreciated $^{30-33}$, and methods to generate this reactive PTM are of interest to the scientific community, especially bioorthogonal methods that avoid the use of non-selective reagents such as $\mathrm{H}_{2} \mathrm{~S}^{26}$. To demonstrate the feasibility of leveraging the thiocysteine lyase activity of the SH domains to install an -SSH group into proteins, we selected regions of GAPDH and NF-kB as two model systems (Fig. 5a, d) ${ }^{32,33}$. We synthesized two pentapeptides, mimicking the regions surrounding the conserved Cys151 and Cys 38 residues that are the sites of sulfhydration within 
GAPDH and NF-kB, respectively, and conjugated them with Lcysteine to prepare the L-thiocysteine adducts 19 and 21 as substrate mimics (Fig. 5b, e). Treatment of 19 and 21 with WsmR-SH resulted in the concentration-dependent formation of new products, the identities of which as the corresponding hydropersulfides were established by analyzing their $\mathrm{mBB}$ adducts 20 and 22 (Supplementary Fig. 25c, d). The SH domains enrich the toolbox of excised PKS domains as biocatalysts for unparalleled chemistry with a broad substrate specificity ${ }^{34-36}$. The biocatalytic strategy provides a promising bioorthogonal solution to the synthesis of biologically relevant hydropersulfides with future opportunities to expand the substrate scope and improve the catalytic efficiency through enzyme evolution.

In conclusion, the LNM family of natural products has served as a great inspiration for the discovery of chemistry, enzymology, biology, and medicine ${ }^{21-24}$. The current study highlights how comparative analysis of the biosynthetic machinery for the LNM family of natural products has provided opportunities to study natural product biosynthesis, leading to the discoveries of a SAMdependent MT that exhibits a high substrate preference for hydropersulfides, thiocysteine lyases as PKS domains that directly install a -SSH group into varying polyketide scaffolds, DUF-SH didomain-containing BGCs widely spread in Nature that extends beyond the biosynthetic machinery for the LNM family of natural products, and $\mathrm{SH}$ domains as biocatalysts that could be leveraged to install an -SSH group into varying biologically relevant scaffolds. These findings set the stage to investigate the catalytic role the DUF domains may play in the formation of the ACPtethered L-thiocysteine-polyketide adducts and the modular architecture and programmability of the DUF-SH domain within the varying type I PKS assembly lines, as well as its portability for engineering polyketide structural diversity. Equally exciting is the continued exploration of the SH domains (as thiocysteine lyases) and GnmP (as a hydropersulfide-specific MT) as biocatalysts, alone to install an -SSH group into biologically relevant scaffolds, or in combination to cap the intrinsically unstable hydropersulfide products as methyl persulfides.

\begin{abstract}
Methods
General experimental procedures. All ${ }^{1} \mathrm{H},{ }^{13} \mathrm{C}$, and $2 \mathrm{D}$ NMR $\left({ }^{1} \mathrm{H}-{ }^{1} \mathrm{H}\right.$ COSY, ${ }^{1} \mathrm{H}-$ ${ }^{13} \mathrm{C}$ HSQC, ${ }^{1} \mathrm{H}_{-}{ }^{13} \mathrm{C}$ HMBC, and ${ }^{1} \mathrm{H}-{ }^{1} \mathrm{H}$ ROESY) experiments were run on a Bruker Avance III Ultrashield 600 at $600 \mathrm{MHz}$ for ${ }^{1} \mathrm{H}$ and $150 \mathrm{MHz}$ for ${ }^{13} \mathrm{C}$ nuclei. NMR data were analyzed using MestReNova 6.0.2-5475. High-performance liquid chromatography (HPLC) was performed on an Agilent 1260 Prep Infinity LC with an MWD detector equipped with an Agilent Eclipse XDB-C18 column $(250 \mathrm{~mm} \times 21.2 \mathrm{~mm}, 7 \mu \mathrm{m})$. Analytical HPLC was performed on an Agilent 1260 Infinity LC with a DAD detector equipped with an Agilent Poroshell 120 EC-C18 column $(250 \mathrm{~mm} \times 4.6 \mathrm{~mm}, 2.7 \mu \mathrm{m})$ with a constant temperature of $35^{\circ} \mathrm{C}$
\end{abstract}

Liquid chromatography (LC)-high-resolution mass spectrometry (MS) analysis. LC-MS was performed on an Agilent 1260 Infinity LC coupled to a 6230 TOF (HRESI) equipped with an Agilent Poroshell 120 EC-C18 column $(50 \mathrm{~mm} \times 4.6 \mathrm{~mm}$, $2.7 \mu \mathrm{m})$ with a constant temperature of $40^{\circ} \mathrm{C}$, using mobile phase $\mathrm{A}(0.1 \%$ formic acid in $\left.\mathrm{H}_{2} \mathrm{O}\right)$ and mobile phase $\mathrm{B}\left(0.1 \%\right.$ formic acid in $\left.\mathrm{CH}_{3} \mathrm{CN}\right)$. Two methods were applied for analysis. Method I was carried out using a 12 min solvent gradient from 5 to $100 \% \mathrm{~B}$ followed with $3 \mathrm{~min}$ of $5 \% \mathrm{~B}$ at a flow rate of $0.4 \mathrm{~mL} \mathrm{~min}^{-1}$. Method II was carried out using a $20 \mathrm{~min}$ solvent gradient from 5 to $100 \%$ B followed with $5 \mathrm{~min}$ of $5 \% \mathrm{~B}$ at a flow rate of $0.4 \mathrm{~mL} \mathrm{~min}^{-1}$. For the deacetylated mycothiol persulfide adduct S15 and its monosaccharide analog S16, as well as other synthetic intermediates, LCMS was performed on a Thermo Vanquish UHPLC coupled to an Orbitrap Exploris 120 (HRESI) equipped with an Accucore C18 column $(100 \mathrm{~mm} \times 2.1 \mathrm{~mm}, 2.6 \mu \mathrm{m})$ with a constant temperature of $35^{\circ} \mathrm{C}$, using mobile phase $\mathrm{A}(0.1 \%$ formic acid in $\left.\mathrm{H}_{2} \mathrm{O}\right)$ and mobile phase $\mathrm{B}\left(0.1 \%\right.$ formic acid in $\left.\mathrm{CH}_{3} \mathrm{CN}\right)$. Samples were eluted with a 5 min solvent gradient from 2 to $98 \%$ B followed with 2 min of $98 \%$ B followed with $2 \mathrm{~min}$ of $2 \% \mathrm{~B}$ at a flow rate of $0.4 \mathrm{~mL} \mathrm{~min}{ }^{-1}$.

Bacterial strains, plasmids, and chemicals. Strains, plasmids, and polymerase chain reaction (PCR) primers used in this study are listed in Supplementary Tables 1-3, respectively. PCR primers were purchased from Sigma-Aldrich. Q5 high-fidelity DNA polymerase, restriction endonucleases, and T4 DNA ligase were purchased from New England Biolabs (NEB) and used following the protocols provided by the manufacturer. DNA gel extraction and plasmid preparation kits were purchased from Omega Bio-Tek. DNA sequencing was conducted by Eton Bioscience. Other chemicals, biochemical, and media components were purchased from standard commercial sources.

Culture conditions. E. coli strains harboring plasmids or cosmids were grown in lysogeny broth (LB) with appropriate antibiotic selection ${ }^{37}$. E. coli ET12567/ pUZ8002 was used for intergeneric conjugation with Streptomyces sp. CB01883 and the conjugations were carried out following standard procedures ${ }^{38}$. Streptomyces sp. CB01883 was cultivated on solid ISP4 medium for sporulation.

Inactivation and complementation of gnmP in Streptomyces sp. CB01883. To construct a plasmid for inactivation of gnmP, a $2063 \mathrm{bp}$ DNA fragment upstream of $g n m P$ was amplified with primers pOJ-1883orf131L-del-F and pOJ1883orf131L-del-R, and a 2099 bp DNA fragment downstream of gnmP was amplified with primers pOJ-1883orf131R2-del-F and pOJ-1883orf131R2-del-R, using cosmid pBS21001 (1C4) of strain CB01883 as a template. The two DNA fragments were digested with the appropriate enzymes and cloned into the HindIII and EcoRI sites of pOJ260 to obtain pBS2101939. Then pBS21019 was transformed into E. coli ET12567 (pUZ8002) and introduced into strain CB01883 by intergeneric conjugation ${ }^{38}$. After several rounds of passaging the exconjugants on solid ISP4 medium, apramycin sensitive mutants were screened by PCR using primers 1883orf131ifdel-2F and 1883orf131ifdel-2R for double-crossover mutants. The genotype of the in-frame deletion mutant strain SB21007 (i.e., $\Delta g n m P$ ) was verified by Southern analysis and PCR (Supplementary Fig. 7).

For the construction of SB21008, a 1219 bp DNA fragment containing gnmP was amplified with primers 1883orf131-kasO-5 and 1883orf131-kasO-3 using cosmid pBS21001 (1C4) as a template and then cloned into the SpeI and EcoRI sites of pBS21003 (pSET-KasO*: constructed by digestion of PCR products obtained with primers PSET-kasO-F and PSET-kasO-R using pSETTurdR as a template with NsiI followed by self-ligation) to obtain pBS21020, in which gnmP was under the control of the strong promoter Kas $O^{* 24,40}$. Then pBS21020 was introduced into SB21007 by intergeneric conjugation to afford the complementation strain SB21008 (i.e., SB21007/gnmP).

Fermentation and identification of GNM-related metabolites. Fresh spores of strain CB01883, its derivative mutant, and complementation strains, were individually inoculated into 250 - $\mathrm{mL}$ baffled flasks containing $50 \mathrm{~mL}$ of TSB seed medium and cultured for $36 \mathrm{~h}$ at $28^{\circ} \mathrm{C}$ and $250 \mathrm{rpm}$. For small-scale fermentations, seed culture was inoculated $(10 \%, \mathrm{v} / \mathrm{v})$ into 250 -mL baffled flasks each containing $50 \mathrm{~mL}$ of production medium (soluble starch $3 \%$, soy flour $1 \%, \mathrm{CaCO}_{3} 0.5 \%, \mathrm{KH}_{2} \mathrm{PO}_{4}$ $0.05 \%, \mathrm{MgSO}_{4} 0.025 \%, \mathrm{ZnSO}_{4} \cdot 7 \mathrm{H}_{2} \mathrm{O} 0.004 \%$, L-methionine $0.01 \%$, vitamin $\mathrm{B}_{12}$ $0.0001 \%, \mathrm{pH} 7.2$ ), a medium modified from the one for the production of LNM by S. atroolivaceus S-14020. Amberlite XAD-16 resin (Sigma) was added to each flask $(4 \%, \mathrm{w} / \mathrm{v})$ at $24 \mathrm{~h}$ after inoculation, and the fermentation was carried out for another $96 \mathrm{~h}$. The resin was harvested from the fermentation broth, washed with water, and allowed to air dry. The dry resin was extracted with $\mathrm{MeOH}(7 \mathrm{~mL})$ and evaporated to dryness. The residue was suspended in $\mathrm{H}_{2} \mathrm{O}(4 \mathrm{~mL})$ and extracted with EtOAc $(7 \mathrm{~mL} \times 2)$. The combined organic phases were evaporated to dryness, and dissolved in $\mathrm{MeOH}(7 \mathrm{~mL})$, which was used directly for LC-MS analysis with Method II

Isolation of GNM-related metabolites S1-S5. For isolation of GNM-related products, a large-scale fermentation $(10 \mathrm{~L})$ of mutant strain SB21007 was performed in a production medium. The resins were harvested and extracted with $\mathrm{MeOH}(2 \mathrm{~L} \times 3)$. The solvent was evaporated to give the crude extract, which was fractionated with MPLC using a Biotage SNAP Ultra C18 $60 \mathrm{~g}$ column with solvent A $\left(0.1 \%\right.$ formic acid in $\left.\mathrm{H}_{2} \mathrm{O}\right)$ and solvent $\mathrm{B}\left(0.1 \%\right.$ formic acid in $\left.\mathrm{CH}_{3} \mathrm{CN}\right)$ as mobile phases. The crude extract was eluted using a solvent gradient from 5 to $100 \% \mathrm{~B}$ over $60 \mathrm{~min}$, followed by $100 \% \mathrm{~B}$ for $5 \mathrm{~min}$ at a flow rate of $30 \mathrm{~mL} \mathrm{~min}{ }^{-1}$. The fractions containing GNM-related compounds were combined based on LC-MS analysis. Combined fractions were concentrated and subjected to a Sephadex LH20 column eluted with methanol. The fractions containing compounds of interest were combined and finally purified by an Agilent HPLC system with a Zorbax SBC13 column $(5 \mu \mathrm{m}, 9.4 \times 250 \mathrm{~mm})$ with solvent $\mathrm{A}\left(0.1 \%\right.$ TFA in $\left.\mathrm{H}_{2} \mathrm{O}\right)$ and solvent B $\left(0.1 \%\right.$ TFA in $\left.\mathrm{CH}_{3} \mathrm{CN}\right)$ as mobile phases. Fraction I was eluted with a linear gradient from 20 to $65 \%$ B over $50 \mathrm{~min}$ at a flow rate of $2 \mathrm{~mL} \mathrm{~min}^{-1}$ to give $\mathbf{S 1}$ $(4.5 \mathrm{mg})$ and $\mathbf{S 2}(3.1 \mathrm{mg})$. Fraction II was purified in an isocratic condition of $45 \%$ B at a flow rate of $2 \mathrm{~mL} \mathrm{~min}{ }^{-1}$ for $40 \mathrm{~min}$ to afford $\mathbf{S 3}(3.3 \mathrm{mg}), \mathbf{S 4}(4.8 \mathrm{mg})$, and $\mathbf{S 5}$ (3.6 mg) (Supplementary Fig. 9).

Gene cloning. To construct a plasmid for production of GnmP from Streptomyces $s p$. CB01883, the region coding gnmP was amplified by PCR from genomic DNA with Q5 DNA polymerase (NEB) following the protocol by the manufacturer using the primers 1883orf131-F and 1883orf131-R. The PCR product was purified, treated with T4 polymerase, and cloned into pBS3080 according to ligationindependent procedures to afford pBS21021. For the construction of plasmids pBS21022 and pBS21023, used to produce GnmT-SH and CB01883-CGL from Streptomyces sp. CB01883, the same procedure was followed with the primers 
GnmT-SH-F and GnmT-SH-R, and CB01883-CGL-F and CB01883-CGL-R, respectively. For the construction of plasmid pBS22010 and pBS22011, used to produce WsmR-SH and CB02120-2-CGL from Streptomyces sp. CB02120-2, the same procedure was followed with the primers WsmR-SH-F and WsmR-SH-R, and CB02120-2-CGL-F and CB02120-2-CGL-R, respectively. For the construction of plasmid, pBS3168 was used to produce CB01635-CGL from Streptomyces sp. CB01635, the same procedure was followed with the primers CB01635-CGL-F and CB01635-CGL-R.

\section{Gene expression and protein production and purification. To overproduce} GnmP, pBS21021 was transformed into E. coli BL21(DE3) (Life Technologies), and the resultant recombinant strain was first grown in $2 \mathrm{~L}$ of $\mathrm{LB}$, containing $50 \mu \mathrm{g} / \mathrm{mL}$ kanamycin, at $37^{\circ} \mathrm{C}$ with shaking at $250 \mathrm{rpm}$. When the $\mathrm{OD}_{600}$ reached 0.6 , the culture was cooled to $4{ }^{\circ} \mathrm{C}$. After the addition of $0.10 \mathrm{mM}$ isopropyl $\beta$-D-1-thiogalactopyranoside (IPTG) to induce gene expression, the culture was then grown overnight at $18{ }^{\circ} \mathrm{C}$ with shaking at $250 \mathrm{rpm}$. To isolate the $\mathrm{N}$-terminal $\mathrm{His}_{6}$-tagged $\mathrm{GnmP}$, the cells were harvested by centrifugation at $4000 \mathrm{~g}$ for $10 \mathrm{~min}$ at $4{ }^{\circ} \mathrm{C}$, resuspended in lysis buffer (100 mM Tris, pH 8.0, containing $300 \mathrm{mM} \mathrm{NaCl}$ $15 \mathrm{mM}$ imidazole, and $10 \%$ glycerol), lysed by sonication, and centrifuged at $15,000 \mathrm{~g}$ for $30 \mathrm{~min}$ at $4{ }^{\circ} \mathrm{C}$ to pellet the cell debris. The supernatant was finally applied to a HisTrap column and purified by nickel affinity chromatography using an ÄKTA FPLC system (GE Healthcare Biosciences). The purified N-terminal $\mathrm{His}_{6}$-tagged GnmP was desalted using a HiPrep desalting column (GE Healthcare Biosciences) in $50 \mathrm{mM}$ Tris buffer, $\mathrm{pH} 7.8$, containing $100 \mathrm{mM} \mathrm{NaCl}$, and $5 \%$ glycerol, and concentrated using an Amicon Ultra-15 concentrator (Millipore) Protein concentrations were determined from the absorbance at $280 \mathrm{~nm}$ using a molar absorptivity constant $\left(\varepsilon_{280}=97,860 \mathrm{M}^{-1} \mathrm{~cm}^{-1}\right)$. GnmT-SH, CB01883-CGL, WsmR-SH, CB02120-2-CGL, and CB01635-CGL were produced and purified with the same procedure. For LnmJ-SH, expression of pBS3109 in E. coli and production and purification of LnmJ-SH to homogeneity followed published procedure ${ }^{22}$.

Analytical size-exclusion chromatography. The molecular weights (MW) and the quaternary state of GnmP, GnmT-SH, LnmJ-SH, WsmR-SH, CB01883-CGL, CB02120-2-CGL, and CB01635-CGL in solution were determined by sizeexclusion chromatography using a Superdex 200 16/600 column (GE Healthcare Life Sciences) connected to an ÄKTAxpress system. For GnmP, the buffer was $20 \mathrm{mM}$ MOPS, pH 7.0, and for GnmT-SH, LnmJ-SH, WsmR-SH, CB01883-CGL, CB02120-2-CGL, and CB01635-CGL, the buffer was $50 \mathrm{mM}$ MOPS, $50 \mathrm{mM} \mathrm{KCl}$, and $100 \mathrm{mM} \mathrm{NaCl}, \mathrm{pH}$ 7.5. The column was pre-equilibrated with two column volumes of buffer, and calibrated with vitamin B12 (1.35 kDa), myoglobin $(17 \mathrm{kDa})$, ovalbumin $(44 \mathrm{kDa}), \gamma$-globulin $(158 \mathrm{kDa})$, and thyroglobin $(670 \mathrm{kDa})$. The chromatography was carried out at $4{ }^{\circ} \mathrm{C}$ at a flow rate of $1 \mathrm{~mL} \min ^{-1}$. The calibration curves of $K_{\mathrm{av}}$ versus $\log (\mathrm{MW})$ for each buffer condition were prepared using the equation $K_{\mathrm{av}}=\left(V_{\mathrm{e}}-V_{\mathrm{o}}\right) /\left(V_{\mathrm{t}}-V_{\mathrm{o}}\right)$, where $V_{\mathrm{e}}, V_{\mathrm{o}}$, and $V_{\mathrm{t}}$ are the elution volume, column void volume, and total bed volume, respectively (Supplementary Figs. 11, 12, and 22a, b).

\section{Enzymatic reactions of GnmT-SH, LnmJ-SH, or WsmR-SH with 16 as a sub-} strate. Each incubation was performed in $50 \mathrm{mM}$ sodium phosphate, $\mathrm{pH} 7.2$, containing $6 \mathrm{mM}$ 16, $50 \mu \mathrm{M}$ GnmT-SH, LnmJ-SH, or WsmR-SH, $0.2 \mathrm{mM}$ PLP, and $20 \mathrm{mM} \mathrm{KCl}$ in a total volume of $50 \mu \mathrm{L}$, respectively. After incubation at $28^{\circ} \mathrm{C}$ for $10 \mathrm{~min}, 100 \mu \mathrm{L}$ of acetonitrile containing $0.1 \% \mathrm{v} / \mathrm{v}$ TFA was added to quench the reaction. The reaction mixture was then centrifuged at $12,000 \mathrm{~g}$ for $10 \mathrm{~min}$. The LC-MS analysis was performed using mobile phase A $\left(0.1 \%\right.$ TFA in $\left.\mathrm{H}_{2} \mathrm{O}\right)$ and mobile phase $\mathrm{B}\left(0.1 \%\right.$ TFA in $\left.\mathrm{CH}_{3} \mathrm{CN}\right)$ with a flow rate of $0.4 \mathrm{~mL} \mathrm{~min}^{-1}$ and a 12 min solvent gradient from 5 to $100 \%$ B followed by 3 min of $5 \%$ B. This allowed direct quantification of substrate $\mathbf{1 6}$ and product $\mathbf{S 1 0}$ in the assay solution, as well as the trace amount of $\mathbf{S 1 1}$ resulted from a retro-Michael reaction upon quenching the assays for analysis (Supplementary Fig. 19).

Formation of $\mathrm{mBB}$-persulfide adducts of the varying hydropersulfide products generated in situ by enzymatic reactions of GnmT-SH, LnmJ-SH, or WsmRSH. For 11 (Fig. 2a, c) or S9 (Supplementary Fig. 18a, b), each incubation was performed in $50 \mathrm{mM}$ sodium phosphate, $\mathrm{pH} 8.0$, containing $0.5 \mathrm{mM} 9$ or $13,10 \mu \mathrm{M}$ GnmT-SH, LnmJ-SH, or WsmR-SH, $0.2 \mathrm{mM}$ PLP, $20 \mathrm{mM} \mathrm{KCl}$, and $0.5 \mathrm{mM} \mathrm{mBB}$ in a total volume of $50 \mu \mathrm{L}$, respectively. After incubation at $28^{\circ} \mathrm{C}$ for $20 \mathrm{~min}, 50 \mu \mathrm{L}$ of $\mathrm{MeOH}$ was added to quench the reaction. The reaction mixture was then centrifuged at $12,000 \mathrm{~g}$ for $10 \mathrm{~min}$ and the supernatant was injected and analyzed by LC-MS with Method I.

For 18 (Fig. 4a, b), each incubation was performed in $50 \mathrm{mM}$ sodium phosphate, $\mathrm{pH} 7.5$, containing $5 \mathrm{mM} \mathrm{17}, 30 \mu \mathrm{M}$ GnmT-SH, LnmJ-SH, or WsmR$\mathrm{SH}, 0.2 \mathrm{mM}$ PLP, $20 \mathrm{mM} \mathrm{KCl}$, and $2 \mathrm{mM} \mathrm{mBB}$ in a total volume of $50 \mu \mathrm{L}$, respectively. After incubation at $28^{\circ} \mathrm{C}$ for $30 \mathrm{~min}, 50 \mu \mathrm{L}$ of methanol was added to quench the reaction. The reaction mixture was then centrifuged at $12,000 \mathrm{~g}$ for $10 \mathrm{~min}$ and the supernatant was injected and analyzed by LC-MS with Method I.

For 20 (Fig. 5a, c) and 22 (Fig. 5d, f), each incubation was performed in $50 \mathrm{mM}$ sodium phosphate, $\mathrm{pH} 7.5$, containing $2 \mathrm{mM} 19$ or $21,30 \mu \mathrm{M}$ GnmT-SH, LnmJSH, or WsmR-SH, $30 \mu \mathrm{M}$ PLP, $20 \mathrm{mM} \mathrm{KCl}, 2 \mathrm{mM} \mathrm{mBB}$, in a total volume of $50 \mu \mathrm{L}$, respectively. After incubation at $28^{\circ} \mathrm{C}$ for $1 \mathrm{~h}, 100 \mu \mathrm{L}$ of methanol was added to quench the reaction. The reaction mixture was then centrifuged at $12,000 \mathrm{~g}$ for $10 \mathrm{~min}$ and the supernatant was injected and analyzed by LC-MS with Method II.

For the deacetylated mycothiol persulfide adduct S15 and its monosaccharide analog S16 as alternative substrate mimics, each incubation was performed in $50 \mathrm{mM}$ Tris, $\mathrm{pH}$ 7.5, containing $1 \mathrm{mM}$ substrate, $30 \mu \mathrm{M}$ GnmT-SH, LnmJ-SH, or WsmR-SH, $0.2 \mathrm{mM}$ PLP, $20 \mathrm{mM} \mathrm{KCl}$, and $1 \mathrm{mM} \mathrm{mBB}$ in a total volume of $50 \mu \mathrm{L}$, respectively, with $\mathbf{1 7}$ as a positive control, affording full conversion to product $\mathbf{1 8}$ After incubation at $28^{\circ} \mathrm{C}$ for $20 \mathrm{~min}, 50 \mu \mathrm{L}$ of $\mathrm{MeOH}$ was added to quench the reaction. The reaction mixture was then centrifuged at $12,000 \mathrm{~g}$ for $10 \mathrm{~min}$ and the supernatant was injected and analyzed by LC-MS with Method I.

\section{Enzymatic formation of $S$-methylated sulfides, disulfides, or trisulfides by} GnmP. For in situ generations of hydropersulfides $\mathbf{7}$ or $\mathbf{8}$ and its subsequent $S$ methylation to afford the $S$-methylated disulfide product 3 (Figs. 2a, c and 3a, b) or 14 (Fig. 3a, c and Supplementary Fig. 18a, c), a GnmT-SH, LnmJ-SH, or WsmR-SH and GnmP-coupled assay was employed using $\mathbf{9}$ or $\mathbf{1 3}$ as a surrogate substrate, respectively. Each incubation was performed in $50 \mathrm{mM}$ sodium phosphate, $\mathrm{pH} 8.0$ containing $0.5 \mathrm{mM} 9$ or $13,10 \mu \mathrm{M}$ GnmT-SH, LnmJ-SH, or WsmR-SH, $0.2 \mathrm{mM}$ PLP, $20 \mathrm{mM} \mathrm{KCl}, 30 \mu \mathrm{M} \mathrm{GnmP}$, and $2 \mathrm{mM} \mathrm{SAM}$ in a total volume of $50 \mu \mathrm{L}$, respectively. After incubation at $28^{\circ} \mathrm{C}$ for $20 \mathrm{~min}, 50 \mu \mathrm{L}$ of methanol was added to quench the reaction. The reaction mixture was then centrifuged at $12,000 \mathrm{~g}$ for $10 \mathrm{~min}$ and the supernatant was injected and analyzed by LC-MS with Method I

For $S$-methylation of $\mathbf{4}$ to afford the $S$-methylated sulfide product $\mathbf{S 8}$ (Supplementary Fig. 16c, e), incubation was performed in $50 \mathrm{mM}$ sodium phosphate, $\mathrm{pH} 8.0,1 \mathrm{mM} \mathrm{4}, 30 \mu \mathrm{M} \mathrm{GnmP}$, and $2 \mathrm{mM}$ SAM in a total volume of $50 \mu \mathrm{L}$. After incubation at $28^{\circ} \mathrm{C}$ for $3 \mathrm{~h}, 50 \mu \mathrm{L}$ of methanol was added to quench the reaction. The reaction mixture was then centrifuged at $12,000 \mathrm{~g}$ for $10 \mathrm{~min}$ and the supernatant was injected and analyzed by LC-MS with Method I.

For $S$-methylation of the hydrotrisulfide, resulted from disproportionation of 7 , to afford the corresponding $S$-methylated trisulfide 12 (Fig. 3a, b), incubation was performed in $50 \mathrm{mM}$ sodium phosphate, $\mathrm{pH} 8.0$, containing $1 \mathrm{mM} \mathrm{9,220 \mu \textrm {M }}$ WsmR-SH, $0.2 \mathrm{mM}$ PLP, $20 \mathrm{mM} \mathrm{KCl}, 10 \mu \mathrm{M} \mathrm{GnmP}$, and $2 \mathrm{mM} \mathrm{SAM}$ in a total volume of $50 \mu \mathrm{L}$. After incubation at $28^{\circ} \mathrm{C}$ for $3 \mathrm{~min}, 250 \mu \mathrm{L}$ of methanol was added to quench the reaction. The reaction mixture was then centrifuged at $12,000 \mathrm{~g}$ for $10 \mathrm{~min}$ and the supernatant was injected and analyzed by LC-MS with Method I.

For production and structural characterization of the $S$-methyl disulfide $\mathbf{1 4}$ and $S$-methyl trisulfide $\mathbf{1 5}$ (Fig. 3a, b), a large-scale enzymatic reaction was performed in $50 \mathrm{mM}$ sodium phosphate, $\mathrm{pH} 8.0$, containing $5 \mathrm{mM} \mathrm{13}, 220 \mu \mathrm{M}$ WsmR-SH, $0.2 \mathrm{mM}$ PLP, $20 \mathrm{mM} \mathrm{KCl}, 30 \mu \mathrm{M} \mathrm{GnmP}$, and $10 \mathrm{mM} \mathrm{SAM}$ in a total volume of $10 \mathrm{ml}$. After incubation at $28^{\circ} \mathrm{C}$ for $2 \mathrm{~h}, 30 \mathrm{ml}$ of methanol was added to quench the reaction. The reaction mixture was then centrifuged at $12,000 \mathrm{~g}$ for $20 \mathrm{~min}$ and the supernatant was injected and analyzed by LC-MS with Method I. The reaction mixture was concentrated, dissolved in $\mathrm{CH}_{3} \mathrm{CN}$, and purified by HPLC. The isolation was conducted using a 35 min solvent gradient from 20 to $100 \% \mathrm{CH}_{3} \mathrm{CN}$ in $\mathrm{H}_{2} \mathrm{O}$ containing $0.1 \%$ formic acid at a flow rate of $3 \mathrm{~mL} \mathrm{~min}^{-1}$ to give $\mathbf{1 4}$ $(3.3 \mathrm{mg})$ and $\mathbf{1 5}(0.3 \mathrm{mg})$.

Monitoring GnmT-SH, LnmJ-SH, or WsmR-SH-catalyzed lyase reactions with the varying substrates by directly measuring the concomitantly produced pyruvate. The direct measurement of pyruvate concomitantly produced from the GnmT-SH, LnmJ-SH, or WsmR-SH-catalyzed reactions with the varying substrates is based on the formation of methylquinoxalinol, after derivatization of the reaction mixture with $o$-phenylenediamine (OPD) ${ }^{41,42}$ (Supplementary Figs. 15, 20, and 23). To $150 \mu \mathrm{L}$ of $12 \mathrm{mM}$ OPD in $3 \mathrm{~N} \mathrm{HCl}$ was added $50 \mu \mathrm{L}$ of reaction mixtures, and resulting mixtures vials were heated to $100^{\circ} \mathrm{C}$ for $30 \mathrm{~min}$. Proteins were precipitated by centrifugation and the supernatant was analyzed by analytical HPLC. HPLC analysis was performed using mobile phase A $(0.1 \%$ formic acid in $\left.\mathrm{H}_{2} \mathrm{O}\right)$ and mobile phase $\mathrm{B}\left(0.1 \%\right.$ formic acid in $\left.\mathrm{CH}_{3} \mathrm{CN}\right)$ with a flow rate of $1 \mathrm{~mL} \mathrm{m^{-1 }}$ and a $20 \mathrm{~min}$ solvent gradient from 5 to $90 \%$ B followed by $6 \mathrm{~min}$ of $100 \% \mathrm{~B}$, unless otherwise indicated. The UV absorption was measured at $340 \mathrm{~nm}$ Standard curves were constructed by mixing $50 \mu \mathrm{L}$ of the pyruvic acid solution instead of the reaction solution with $150 \mu \mathrm{L}$ of $12 \mathrm{mM}$ OPD in $3 \mathrm{~N} \mathrm{HCl}$ with pyruvate concentrations ranging from 12.5 to $125 \mu \mathrm{M}$.

Kinetic studies of GnmT-SH, LnmJ-SH, or WsmR-SH catalysis with 9, 16, 17, 19, or 21 as a substrate. For kinetics studies of GnmT-SH, LnmJ-SH, or WsmRSH catalysis with 9 as a substrate, each incubation was performed in $50 \mathrm{mM}$ sodium phosphate, $\mathrm{pH}$ 8.0, (Tris-HCl, $\mathrm{pH} 8.5$ for LnmJ-SH), $0.5 \mu \mathrm{M}$ GnmT-SH, LnmJ-SH, or WsmR-SH, $0.2 \mathrm{mM} \mathrm{PLP}$, and $20 \mathrm{mM} \mathrm{KCl}$, with substrate concentrations varied from $0.76 \mathrm{mM}$ to $9.12 \mathrm{mM}$, in a total volume of $50 \mu \mathrm{L}$, respectively. After incubation at $28^{\circ} \mathrm{C}$ for $100 \mathrm{~min}, 150 \mu \mathrm{L}$ of $12 \mathrm{mM}$ OPD in $3 \mathrm{~N} \mathrm{HCl}$ was added to the reaction, and the resulting mixtures vials were heated to $100^{\circ} \mathrm{C}$ for $30 \mathrm{~min}$. The reaction mixture was then centrifuged at $12,000 \mathrm{~g}$ for $10 \mathrm{~min}$, and the supernatant was injected and analyzed by HPLC with the above method (Supplementary Fig. 15).

For kinetics studies of GnmT-SH, LnmJ-SH, or WsmR-SH with $\mathbf{1 6}$ as a substrate, each incubation was performed in $50 \mathrm{mM}$ sodium phosphate, $\mathrm{pH} 7.2$, containing $5 \mu \mathrm{M}$ GnmT-SH, LnmJ-SH, or WsmR-SH, $0.2 \mathrm{mM}$ PLP, and $20 \mathrm{mM}$ 
$\mathrm{KCl}$, with substrate concentrations varied from $1.25 \mathrm{mM}$ to $150 \mathrm{mM}$, in a total volume of $50 \mu \mathrm{L}$, respectively. After incubation at $28^{\circ} \mathrm{C}$ for $10 \mathrm{~min}, 150 \mu \mathrm{L}$ of $12 \mathrm{mM}$ OPD in $3 \mathrm{~N} \mathrm{HCl}$ was added to the reaction, and the resulting mixtures vials were heated to $100^{\circ} \mathrm{C}$ for $30 \mathrm{~min}$. The reaction mixture was then centrifuged at $12,000 \mathrm{~g}$ for $10 \mathrm{~min}$, and the supernatant was injected and analyzed by HPLC with the above method (Supplementary Fig. 20a-e).

For kinetics studies of GnmT-SH, LnmJ-SH, or WsmR-SH with $\mathbf{1 7}$ as a substrate, each incubation was performed in $50 \mathrm{mM}$ sodium phosphate, $\mathrm{pH} 7.2$, containing $5 \mu \mathrm{M}$ GnmT-SH, LnmJ-SH, or WsmR-SH, $0.2 \mathrm{mM}$ PLP, and $20 \mathrm{mM}$ $\mathrm{KCl}$, with substrate concentrations varied from 1.25 to $80 \mathrm{mM}$, in a total volume of $50 \mu \mathrm{L}$. After incubation at $28^{\circ} \mathrm{C}$ for $10 \mathrm{~min}, 150 \mu \mathrm{L}$ of $12 \mathrm{mM}$ OPD in $3 \mathrm{~N} \mathrm{HCl}$ was added to the reaction, and the resulting mixtures vials were heated to $100^{\circ} \mathrm{C}$ for $30 \mathrm{~min}$. The reaction mixture was then centrifuged at $12,000 \mathrm{~g}$ for $10 \mathrm{~min}$, and the supernatant was injected and analyzed by HPLC with the above method (Supplementary Fig. 20a, b, f-h).

For kinetics studies of WsmR-SH with 19 as a substrate, each incubation was performed in $50 \mathrm{mM}$ sodium phosphate, $\mathrm{pH} 7.5$, containing $30 \mu \mathrm{M}$ WsmR-SH, $0.2 \mathrm{mM}$ PLP, and $20 \mathrm{mM} \mathrm{KCl}$, with substrate concentrations varied from $0.306 \mathrm{mM}$ to $10 \mathrm{mM}$, in a total volume of $25 \mu \mathrm{L}$. After incubation at $28^{\circ} \mathrm{C}$ for $10 \mathrm{~min}, 75 \mu \mathrm{L}$ of $12 \mathrm{mM}$ OPD in $3 \mathrm{~N} \mathrm{HCl}$ was added to the reaction, and the resulting mixtures were heated to $100^{\circ} \mathrm{C}$ for $30 \mathrm{~min}$. The reaction mixture was then centrifuged at $12,000 \mathrm{~g}$ for $10 \mathrm{~min}$, and the supernatant was injected and analyzed by HPLC. HPLC analysis was performed using mobile phase $\mathrm{A}\left(0.1 \%\right.$ formic acid in $\left.\mathrm{H}_{2} \mathrm{O}\right)$ and mobile phase $\mathrm{B}\left(0.1 \%\right.$ formic acid in $\left.\mathrm{CH}_{3} \mathrm{CN}\right)$ with a flow rate of $1 \mathrm{~mL} \mathrm{~min}{ }^{-1}$ and a 40 min solvent gradient from 5 to $95 \%$ B followed by 6 min of $100 \%$ B. The UV absorption was measured at $340 \mathrm{~nm}$ (Supplementary Fig. 25a, b, e).

For kinetics studies of WsmR-SH with $\mathbf{2 1}$ as a substrate, each incubation was performed in $50 \mathrm{mM}$ sodium phosphate, $\mathrm{pH} 7.5$, containing $30 \mu \mathrm{M}$ WsmR-SH, $0.2 \mathrm{mM} \mathrm{PLP}$, and $20 \mathrm{mM} \mathrm{KCl}$, with substrate concentrations varied from $0.375 \mathrm{mM}$ to $20 \mathrm{mM}$, in a total volume of $25 \mu \mathrm{L}$. After incubation at $28^{\circ} \mathrm{C}$ for $10 \mathrm{~min}, 75 \mu \mathrm{L}$ of $12 \mathrm{mM}$ OPD in $3 \mathrm{~N} \mathrm{HCl}$ was added to the reaction, and the resulting mixtures were heated to $100^{\circ} \mathrm{C}$ for $30 \mathrm{~min}$. The reaction mixture was then centrifuged at $12,000 \mathrm{~g}$ for $10 \mathrm{~min}$, and the supernatant was injected and analyzed by HPLC with the above method given for substrate 19 (Supplementary Fig. 25a, b, f).

Kinetic studies of GnmP with $\mathbf{7}$ or $\mathbf{4}$ as a substrate. For kinetics studies of GnmP with 7 as a substrate, generated in situ from 9, each incubation was performed in $50 \mathrm{mM}$ sodium phosphate, $\mathrm{pH} 8.0$, containing $220 \mu \mathrm{M}$ WsmR-SH, $0.2 \mathrm{mM}$ PLP, $20 \mathrm{mM} \mathrm{KCl}, 0.05 \mu \mathrm{M} \mathrm{GnmP}$, and $2 \mathrm{mM} \mathrm{SAM}$, with substrate concentrations varied from 1.25 to $25 \mathrm{mM}$, in a total volume of $50 \mu \mathrm{L}$, respectively. After incubation at $28^{\circ} \mathrm{C}$ for $3.5 \mathrm{~min}, 100 \mu \mathrm{L}$ of methanol was added to quench the reaction. The reaction mixture was then centrifuged at $12,000 \mathrm{~g}$ for $20 \mathrm{~min}$, and the supernatant was injected and analyzed by HPLC. HPLC analysis was performed using mobile phase A $\left(0.1 \%\right.$ formic acid in $\left.\mathrm{H}_{2} \mathrm{O}\right)$ and mobile phase $\mathrm{B}(0.1 \%$ formic acid in $\mathrm{CH}_{3} \mathrm{CN}$ ) with a flow rate of $1 \mathrm{~mL} \mathrm{~min}^{-1}$ and a $30 \mathrm{~min}$ solvent gradient from 5 to $90 \%$ B followed by 5 min of $100 \%$ B (Supplementary Fig. 16a, b).

For kinetics studies of $\mathrm{GnmP}$ with $\mathbf{4}$ as a substrate, each incubation was performed in $50 \mathrm{mM}$ sodium phosphate, $\mathrm{pH} 8.0$, containing $2 \mathrm{mM}$ SAM, with substrate concentrations varied from 10 to $400 \mu \mathrm{M}$, and $2.5 \mu \mathrm{M} \mathrm{GnmP}$ in a total volume of $50 \mu \mathrm{L}$. After incubation at $28^{\circ} \mathrm{C}$ for $200 \mathrm{~min}, 100 \mu \mathrm{L}$ of methanol was added to quench the reaction. The reaction mixture was then centrifuged at $12,000 \mathrm{~g}$ for $20 \mathrm{~min}$, and the supernatant was injected and analyzed by HPLC. HPLC analysis was performed using mobile phase A $\left(0.1 \%\right.$ formic acid in $\left.\mathrm{H}_{2} \mathrm{O}\right)$ and mobile phase $\mathrm{B}\left(0.1 \%\right.$ formic acid in $\left.\mathrm{CH}_{3} \mathrm{CN}\right)$ with a flow rate of $1 \mathrm{~mL} \mathrm{~min}-1$ and a $40 \mathrm{~min}$ solvent gradient from 5 to $90 \%$ B followed by $10 \mathrm{~min}$ of $100 \% \mathrm{~B}$ (Supplementary Fig. 16c-f).

\section{Formation of the $\mathrm{mBB}$-thiocysteine adduct of thiocysteine generated in a suit} by enzymatic reactions of CB01883-CGL, CB02120-2-CGL, or CB01635-CGL with L-cystine as a substrate. As L-cystine is notoriously insoluble, two equivalents of $\mathrm{KOH}$ were used with gentle heating and sonication to obtain a $0.1 \mathrm{M}$ solution. Each incubation was performed in $50 \mathrm{mM}$ sodium phosphate, $\mathrm{pH} 8.0$, containing $10 \mathrm{mM}$ cystine, $50 \mu \mathrm{M}$ CB01883-CGL, CB02120-2-CGL, or CB01635-CGL, $0.2 \mathrm{mM}$ PLP, and $20 \mathrm{mM} \mathrm{KCl}$ in a total volume of $50 \mu \mathrm{L}$, respectively. After incubation at $28^{\circ} \mathrm{C}$ for $5 \mathrm{~min}, 50 \mu \mathrm{L}$ of acetonitrile was added to quench the reaction. Subsequently, $1 \mu \mathrm{L}$ of $100 \mathrm{mM} \mathrm{mBB}$ in acetonitrile was added to the quench reaction mixture, and incubation was conducted at room temperature for $5 \mathrm{~min}$ in the dark. The reaction mixture was then centrifuged at $12,000 \mathrm{~g}$ for $10 \mathrm{~min}$. The LC-MS analysis was performed using mobile phase A $(0.1 \%$ TFA in $\mathrm{H} 2 \mathrm{O})$ and mobile phase $\mathrm{B}(0.1 \%$ TFA in $\mathrm{CH}_{3} \mathrm{CN}$ ) with a flow rate of $0.4 \mathrm{~mL} \mathrm{~min}^{-1}$ and a 12 min solvent gradient from 5 to $100 \%$ B followed by 3 min of 5\% B (Supplementary Fig. 23a-e, g).

\section{Kinetic studies of CB01883-CGL, CB01635-CGL, or CB02120-2-CGL with} cystine as a substrate. For kinetics studies of CB01883-CGL, CB02120-2-CGL, or CB01635-CGL with cystine as a substrate, each incubation was performed in $50 \mathrm{mM}$ sodium phosphate, $\mathrm{pH} 8.0$ (sodium phosphate, $\mathrm{pH} 7.5$ for WSM-CGL), $10 \mu \mathrm{M}$ CB01883-CGL, CB02120-2-CGL, or CB01635-CGL, 0.2 mM PLP, and $20 \mathrm{mM} \mathrm{KCl}$, with substrate concentrations varied from 3 to $10 \mathrm{mM}$, in a total volume of $50 \mu \mathrm{L}$, respectively. After incubation at $28^{\circ} \mathrm{C}$ for $15 \mathrm{~min}, 150 \mu \mathrm{L}$ of
$12 \mathrm{mM}$ OPD in $3 \mathrm{~N} \mathrm{HCl}$ was added to the reaction, and heated to $100^{\circ} \mathrm{C}$ for $30 \mathrm{~min}$. The reaction mixture was then centrifuged at $12,000 \mathrm{~g}$ for $10 \mathrm{~min}$, and the supernatant was analyzed by HPLC. HPLC analysis was performed using mobile phase A $\left(0.1 \%\right.$ formic acid in $\left.\mathrm{H}_{2} \mathrm{O}\right)$ and mobile phase $\mathrm{B}(0.1 \%$ formic acid in $\mathrm{CH}_{3} \mathrm{CN}$ ) with a flow rate of $1 \mathrm{~mL} \mathrm{~min}^{-1}$ and a 20 min solvent gradient from 5 to $90 \%$ B followed by 6 min of 100\% B (Supplementary Fig. 23f).

Statistics and reproducibility. All enzyme assays and protein analysis experiments were verified with at least two independent enzyme preparations. Data are presented as mean values \pm S.E.M. and error bars represent S.E.M. values.

Reporting summary. Further information on research design is available in the Nature Research Reporting Summary linked to this article.

\section{Data availability}

All DNA sequence data used are publicly available: the LNM BGC sequence from $S$. atroolivaceous S-140 is accessible via GenBank under accession number AF484556, the GNM BGC sequence from S. sp. CB01883 under MF925481, and WSM BGC sequence from S. sp. CB02120-2 under MF925482. The raw data used for Supplementary Figs. 7, 11,12 , and 22 are provided in the Source Data file. All other data that support the findings of this study are available in the manuscript and the Supplementary Information. Source data are provided with this paper.

Received: 22 April 2021; Accepted: 1 September 2021; Published online: 28 September 2021

\section{References}

1. Bagley, M. C., Dale, J. W., Merritt, E. A. \& Xiong, X. Thiopeptide antibiotics. Chem. Rev. 105, 685-714 (2005).

2. Dunbar, K. L., Scharf, D. H., Litomska, A. \& Hertweck, C. Enzymatic carbonsulfur bond formation in natural product biosynthesis. Chem. Rev. 117, 5521-5577 (2017).

3. Jiang, C.-S., Müller, W. E. G., Schröder, H. C. \& Guo, Y.-W. Disulfide- and multisulfide-containing metabolites from marine organisms. Chem. Rev. 112 2179-2207 (2012).

4. Jiménez, C. Marine sulfur-containing natural products. (ed R. Atta ur) Studies in Natural Products Chemistry. (Elsevier, 2001), vol. 25, pp. 811-917.

5. Prinsep, M. R. Sulfur-containing natural products from marine invertebrates (ed A.-U. Rahman) Studies in Natural Products Chemistry. (Elsevier, 2003), vol. 28, pp. 617-751.

6. Bewley, K. D. et al. Capture of micrococcin biosynthetic intermediates reveals C-terminal processing as an obligatory step for in vivo maturation. Proc. Natl Acad. Sci. USA 113, 12450-12455 (2016).

7. Hudson, G. A., Zhang, Z., Tietz, J. I., Mitchell, D. A. \& van der Donk, W. A. In vitro biosynthesis of the core scaffold of the thiopeptide thiomuracin. J. Am. Chem. Soc. 137, 16012-16015 (2015).

8. Lombó, F. et al. Deciphering the biosynthesis pathway of the antitumor thiocoraline from a marine actinomycete and its expression in two Streptomyces species. ChemBioChem 7, 366-376 (2006).

9. Sato, M., Nakazawa, T., Tsunematsu, Y., Hotta, K. \& Watanabe, K. Echinomycin biosynthesis. Curr. Opin. Chem. Biol. 17, 537-545 (2013).

10. Zhao, Q., Wang, M., Xu, D., Zhang, Q. \& Liu, W. Metabolic coupling of two small-molecule thiols programs the biosynthesis of lincomycin A. Nature 518, 115-119 (2015)

11. Song, H., Leninger, M., Lee, N. \& Liu, P. Regioselectivity of the oxidative C-S bond formation in ergothioneine and ovothiol biosyntheses. Org. Lett. 15, 4854-4857 (2013).

12. Scharf, D. H. et al. Epidithiol formation by an unprecedented twin carbonsulfur lyase in the gliotoxin pathway. Angew. Chem. Int. Ed. Engl. 51, 10064-10068 (2012)

13. Sasaki, E. et al. Co-opting sulphur-carrier proteins from primary metabolic pathways for 2-thiosugar biosynthesis. Nature 510, 427-431 (2014).

14. Tang, M. C., Zou, Y., Watanabe, K., Walsh, C. T. \& Tang, Y. Oxidative cyclization in natural product biosynthesis. Chem. Rev. 117, 5226-5333 (2017).

15. Scharf, D. H. et al. Flavoenzyme-catalyzed formation of disulfide bonds in natural products. Angew. Chem. Int. Ed. Engl. 53, 2221-2224 (2014).

16. Jacob, C. A scent of therapy: pharmacological implications of natural products containing redox- active sulfur atoms. Nat. Prod. Rep. 23, 851-863 (2006)

17. Wang, C., Wesener, S. R., Zhang, H. \& Cheng, Y. Q. An FAD-dependent pyridine nucleotide-disulfide oxidoreductase is involved in disulfide bond formation in FK228 anticancer depsipeptide. Chem. Biol. 16, 585-593 (2009).

18. Li, B. \& Walsh, C. T. Streptomyces clavuligerus $\mathrm{HlmI}$ is an intramolecular disulfide-forming dithiol oxidase in holomycin biosynthesis. Biochemistry $\mathbf{5 0}$, $4615-4622$ (2011) 
19. Scharf, D. H. et al. Epidithiodiketopiperazine biosynthesis: a four-enzyme cascade converts glutathione conjugates into transannular disulfide bridges. Angew. Chem. Int. Ed. Engl. 52, 11092-11095 (2013).

20. Hara, M. et al. Leinamycin, a new antitumor antibiotic from Streptomyces; producing organism, fermentation and isolation. J. Antibiot. 42, 1768-1774 (1989).

21. Tang, G.-L., Cheng, Y. Q. \& Shen, B. Leinamycin biosynthesis revealing unprecedented architectural complexity for a hybrid polyketide synthase and nonribosomal peptide synthetase. Chem. Biol. 11, 33-45 (2004).

22. Ma, M., Lohman, J. R., Liu, T. \& Shen, B. C-S bond cleavage by a polyketide synthase domain. Proc. Natl Acad. Sci. USA 112, 10359-10364 (2015).

23. Huang, S.-X. et al. Leinamycin E1 acting as an anticancer prodrug activated by reactive oxygen species. Proc. Natl Acad. Sci. USA 112, 8278-8283 (2015).

24. Pan, G. et al. Discovery of the leinamycin family of natural products by mining actinobacterial genomes. Proc. Natl Acad. Sci. USA 114, E11131-E11140 (2017).

25. Becerril, A. et al. Discovery of cryptic largimycins in Streptomyces reveals novel biosynthetic avenues enriching the structural diversity of the leinamycin family. ACS Chem. Biol. 15, 1541-1553 (2020).

26. Park, C. M., Weerasinghe, L., Day, J. J., Fukuto, J. M. \& Xian, M. Persulfides: current knowledge and challenges in chemistry and chemical biology. Mol. Biosyst. 11, 1775-1785 (2015).

27. Nagasawa, T., Kanzaki, H. \& Yamada, H. Cystathionine $\gamma$-lyase of Streptomyces phaeochromogenes. The occurrence of cystathionine $\gamma$-lyase in filamentous bacteria and its purification and characterization. J. Biol. Chem 259, 10393-10403 (1984)

28. Newton, G. L. et al. Distribution of thiols in microorganisms: mycothiol is a major thiol in most Actinomycetes. J. Bacteriol. 178, 1990-1995 (1996).

29. Koledin, T., Newton, G. L. \& Fahey, R. C. Identification of the mycothiol synthase gene $(m s h D)$ encoding the acetyltransferase producing mycothiol in actinomycetes. Arch. Microbiol. 178, 331-337 (2002).

30. Mustafa, A. K. et al. $\mathrm{H}_{2} \mathrm{~S}$ signals through protein S-sulfhydration. Sci. Signal. 2, ra72 (2009).

31. Aroca, A., Serna, A., Gotor, C. \& Romero, L. C. S-sulfhydration: a cysteine posttranslational modification in plant systems. Plant Physiol. 168, 334-342 (2015).

32. Mir, S., Sen, T. \& Sen, N. Cytokine-induced GAPDH sulfhydration affects PSD95 degradation and memory. Mol. Cell 56, 786-795 (2014).

33. Sen, N. et al. Hydrogen sulfide-linked sulfhydration of NF- $\mathrm{BB}$ mediates its antiapoptotic actions. Mol. Cell 45, 13-24 (2012).

34. Piasecki, S. K. et al. Employing modular polyketide synthase ketoreductases as biocatalysts in the preparative chemoenzymatic syntheses of diketide chiral building blocks. Chem. Biol. 18, 1331-1340 (2011).

35. Hansen, D. A. et al. Biocatalytic synthesis of pikromycin, methymycin, neomethymycin, novamethymycin, and ketomethymycin. J. Am. Chem. Soc 135, 11232-11238 (2013).

36. Østergaard, L. H. et al. Stereochemistry of catalysis by the ketoreductase activity in the first extension module of the erythromycin polyketide synthase. Biochemistry 41, 2719-2726 (2002).

37. Sambrook, J. \& Russell, D. W. Molecular cloning: a laboratory manual (3rd edn.). (Cold Spring Harbor Laboratory Press, New York, 2001).

38. Kieser, T., Bibb, M. J., Buttner, M. J., Chater, K. F. \& Hopwood D. A. Practical Streptomyces Genetics. (The John Innes Foundation, Norwich, 2000).

39. Bierman, M. et al. Plasmid cloning vector for the conjugal transfer of DNA from Escherichia coli to Streptomyces spp. Gene 116, 43-49 (1992).

40. Wang, W. et al. An engineered strong promoter for Streptomycetes. Appl. Environ. Microbiol. 79, 4484-4492 (2013).
41. Stijntjes, G. J., te Koppele, J. M. \& Vermeulen, N. P. High-performance liquid chromatography fluorescence assay of pyruvic acid to determine cysteine conjugate $\beta$-lyase activity: application to $S$-1,2-dichlorovinyl-L-cysteine and $S$ 2-benzothiazolyl-L-cysteine. Anal. Biochem. 206, 334-343 (1992).

42. Koike, K. \& Koike, M. Fluorescent analysis of $\alpha$-keto acids in serum and urine by high-performance liquid chromatography. Anal. Biochem. 141, 481-487 (1984).

\section{Acknowledgements}

We thank Kyowa Hakko Kogyo Co. Ltd., Japan for the wild-type S. atroolivaceus S-140 strain. This work was supported in part by NIH grant GM134954 (B.S.). A.D.S. and E.K. are supported in part by NIH Postdoctoral fellowships GM133114 and GM134688, respectively, and W.Y. is supported in part by the Nanjing Agricultural University, Nanjing, China and a scholarship from the Chinese Scholarship Council. This is paper \#30035 from The Scripps Research Institute.

\section{Author contributions}

B.S. conceived the project, S.M., A.D.S., W.Y. and B.S. designed the experiments, S.M., A.D.S., W.Y., G.P., E.K., Y.-C.L. and Z.X. executed the experiments, S.M., A.D.S., W.Y., E.K., Y.-C.L. and B.S. analyzed the results, and S.M., A.D.S., W.Y. and B.S. wrote the paper with inputs from all co-authors.

\section{Competing interests}

The authors declare no competing interests.

\section{Additional information}

Supplementary information The online version contains supplementary material available at https://doi.org/10.1038/s41467-021-25798-8.

Correspondence and requests for materials should be addressed to Ben Shen.

Peer review information Nature Communications thanks the anonymous reviewer(s) for their contribution to the peer review of this work. Peer reviewer reports are available.

Reprints and permission information is available at http://www.nature.com/reprints

Publisher's note Springer Nature remains neutral with regard to jurisdictional claims in published maps and institutional affiliations.

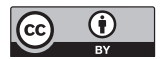

Open Access This article is licensed under a Creative Commons Attribution 4.0 International License, which permits use, sharing, adaptation, distribution and reproduction in any medium or format, as long as you give appropriate credit to the original author(s) and the source, provide a link to the Creative Commons license, and indicate if changes were made. The images or other third party material in this article are included in the article's Creative Commons license, unless indicated otherwise in a credit line to the material. If material is not included in the article's Creative Commons license and your intended use is not permitted by statutory regulation or exceeds the permitted use, you will need to obtain permission directly from the copyright holder. To view a copy of this license, visit http://creativecommons.org/ licenses/by/4.0/.

(c) The Author(s) 2021 